\title{
Hippocampal granule cell dispersion: a non-specific finding in pediatric patients with no history of seizures
}

\author{
Achira Roy ${ }^{1}$, Kathleen J. Millen ${ }^{1,2}$ and Raj P. Kapur ${ }^{1,3,4^{*}}$
}

\begin{abstract}
Chronic epilepsy has been associated with hippocampal abnormalities like neuronal loss, gliosis and granule cell dispersion. The granule cell layer of a normal human hippocampal dentate gyrus is traditionally regarded as a compact neuron-dense layer. Histopathological studies of surgically resected or autopsied hippocampal samples primarily from temporal lobe epilepsy patients, as well as animal models of epilepsy, describe variable patterns of granule cell dispersion including focal cell clusters, broader thick segments, and bilamination or "tram-tracking". Although most studies have implicated granule cell dispersion as a specific feature of chronic epilepsy, very few "non-seizure" controls were included in these published investigations. Our retrospective survey of 147 cadaveric pediatric human hippocampi identified identical morphological spectra of granule cell dispersion in both normal and seizure-affected brains. Moreover, sections across the entire antero-posterior axis of a control cadaveric hippocampus revealed repetitive occurrence of different morphologies of the granule cell layer - compact, focally disaggregated and bilaminar. The results indicate that granule cell dispersion is within the spectrum of normal variation and not unique to patients with epilepsy. We speculate that sampling bias has been responsible for an erroneous dogma, which we hope to rectify with this investigation.
\end{abstract}

Keywords: Hippocampus, Granule cell dispersion, Human, Epilepsy, Dentate gyrus, Temporal lobe epilepsy, Gliosis

\section{Introduction}

Chronic epilepsy is often associated with several pathological hippocampal abnormalities [44]. These include sclerosis, characterized by neuronal loss in both dentate gyrus and Ammon's horn, gliosis, mossy fiber sprouting; as well as granule cell dispersion (GCD), which is observed in the presence or absence of sclerosis $[9,10,15$, $30,55,56]$. These studies were mostly performed in surgical resections or autopsied brain samples obtained from patients suffering from febrile seizures and temporal lobe epilepsy (TLE); it is unclear whether the

\footnotetext{
* Correspondence: raj.kapur@seattlechildrens.org

${ }^{1}$ Center for Integrative Brain Research, Seattle Children's Research Institute, Seattle, Washington, USA

${ }^{3}$ Department of Pathology, Seattle Children's Hospital, Seattle, Washington, USA Full list of author information is available at the end of the article
}

putative hippocampal abnormalities are the cause or effect of epilepsy [14, 25].

GCD has been reported commonly in the dentate gyrus (DG) of seizure patients and considered a hallmark of chronic epilepsy, especially TLE [3, 21, 30, 35, 55, 56]. GCD was also reported to be pronounced in sudden unexplained deaths in infants [26, 31]. Many studies using animal models have similarly concluded that hippocampal GCD is a specific feature of chronic epilepsy [40, 43, 60]. The granule cell (GC) layer is generally observed as a highly compact layer of neuronal cell bodies in the dentate gyrus. GCD refers to broadening with or without bilamination of the GC layer, often blurring the boundary between the GC layer and molecular layer of the dentate gyrus $[8,17]$. Additionally, some surgical cases also demonstrated the existence of focal clusters of 
granule cells outside the regular GC layer [54]. GCD has been most consistently correlated with a history of severe febrile or infection-induced seizures during early life $(<4$ years age), as well as with duration and frequency of epileptic events [30, 36, 54].

Criteria for determining dispersion vary and depend, in part, on the mode of measurement [8]. A variety of diagnostic criteria for GCD has been suggested, varying from complex morphometric analyses [24, 37] to subjective assessment of dentate gyrus histology $[1,8,9,35$, 36]. Some reports have suggested abnormal neuronal migration, loss of hilar cells and genetic defects to be responsible for GCD, all influenced by seizure occurrence $[10,30,54]$. However, a substantial cohort of controls, with no history of seizures, was seldom included in the clinical studies; many lacked even 1 control specimen.

Although most reports claim GCD to be a unique feature of epileptic brains, occasional contradictions in either the identification or interpretation of GCD have been published. The first such report identified GCD in two neurologically normal (non-seizure) patients [25]. They suggested that the pathological changes in the DG are not exclusively related to epilepsy, and speculated that GCD may be a separate developmental disorder, independent of TLE $[8,25]$. Another study suggested that GCD is not a common result of recurring seizure occurrences originating at an early age, but is more dependent on the type of epileptic syndrome [37]. Finally, there are conflicting data on whether GCD in epileptic patients is due to ectopic GC neurogenesis $[6,20]$. At present, there is no consensus over the clinical relevance of GCD or its role in surgical prognosis $[8,9]$.

We began to question the association between seizures and GCD in the hippocampus, after the phenomenon was observed in cadaveric hippocampi of non-seizure patients. In order to better understand GCD and its clinical correlates, we conducted a retrospective assessment of 147 cadaveric pediatric hippocampi (21 epileptic cases, 126 controls with no history of seizure), with a focus exclusively on the DG. We based our study on the hypothesis that GCD is not specifically associated with epilepsy and may be a variant of normal or a nonspecific alteration associated with a wide variety of brain insults.

\section{Materials and methods}

\section{Human hippocampal samples and clinical records}

After appropriate approval from the Institutional Review Board, 147 archived H\&E-stained, coronal sections of hippocampus and associated clinical history were obtained from pediatric patients autopsied at Seattle Children's Hospital between (2014-2019). 126 controls (C, no history of seizures) and 21 seizure (SZ) cases were included in this retrospective study. The following patient- related criteria were abstracted from the archived records: age, gender, post-mortem interval (i.e. duration between time of death and that of autopsy), presence or absence of seizure history (and seizure interval from onset until death for epileptic patients) and major clinical or pathological diagnoses. Corrected age was calculated considering 40 gestational weeks $(\mathrm{GW})$ as day 0 . Among the 147 autopsy cases studied, 80 were males and 67 were females, with the corrected age range spanning from $19 \mathrm{GW}$ (i.e. -21 weeks) to 20.4 years (i.e. +1060 weeks). Post-mortem interval (PMI) ranged from 2 to $336 \mathrm{~h}$.

\section{Histological assessment of GCD}

Presence or absence of GCD (disaggregated, tram-track) was determined by analyzing hematoxylin-and-eosin (H\&E)-stained coronal human hippocampal sections. Archived coronal sections of hippocampus, which were originally prepared as part of routine autopsy examination, were screened retrospectively by a single observer (AR). All available sections (298 sections from 147 patients) were reviewed. For most of the patients, this consisted of a single section (Control: 79/126, 62.70\%; Seizure: $12 / 21,57.14 \%$ ); two sections were available for 26 controls $(20.63 \%)$ and 2 seizure cases $(9.52 \%)$, and three or more sections for the remainder (Control: 21/ 126, 16.67\%; Seizure: 7/21, 33.33\%). Criteria for diagnosis of GCD were the following, irrespective of whether the interfaces between the DG and the hilus or molecular layer were sharp versus diffuse:

(a) "Disaggregated" GCD: focal broadening of the GC layer (>120 $\mu \mathrm{m}$ thickness) without any gaps,

(b) "Tram-track" GCD: focal bilamination of the GC layer with a distinct cell-sparse zone between the inner and outer layers.

In our study, we encountered a few cases with small clusters of "ectopic" granule cells in the hilar region (2 seizure cases, 2 controls) or in the DG molecular layer (1 control, 1 seizure case). These ectopic clusters were always associated with TT or DA.

Any cases deemed initially as definitive or equivocal GCD were re-examined by two observers (AR and RPK) to arrive at a consensus diagnosis. No hippocampal section was excluded from our analysis. Presence versus absence of GCD, along with GCD subtyping, was based on pooled data obtained from all hippocampal sections of a single patient, including sections from both hippocampi when available. For one control (C-20), the entire hippocampus was divided coronally into serial tissue slabs (each $\sim 5 \mathrm{~mm}$ thick) along the antero-posterior axis. Hematoxylin-eosin (H\&E)-stained sections from each slab were examined. 


\section{Immunohistochemistry}

Immunohistochemistry and immunofluorescence were performed on $\sim 10$ control brains and 6 epileptic brains, chosen based on preliminary tissue histology and sufficient tissue availability. The chosen cases represent a wide range of overlapping ages in the patient and control cohorts (Supplementary Table 1, Additional File 1). Paraffin-embedded, formalin-fixed, hippocampal tissue blocks ( 4-5 $\mathrm{mm}$ thick) were obtained and sectioned at average thickness of $5 \mu \mathrm{m}$. Sections were then deparaffinized, as mentioned in the next section, and immunolabelled with mouse anti-CD163 (Leica, Germany; RRID:AB_2756375), rabbit anti-SOX2 (Invitrogen, USA; RRID:AB 2539862), goat anti-PROX1 (R\&D systems, USA; RRID:AB_2170716), mouse anti-human CD68 (Dako, Denmark; RRID:AB 2074844) and mouse anti-GFAP (Dako, Denmark; RRID:AB_2109952) using a Ventana Benchmark II automated immunostainer. PROX1 immunolabeling needed extra stringent conditions: treatment with citrate buffer (20 mins, steamer) and overnight antibody incubation at $4{ }^{\circ} \mathrm{C}$. Other antibodies were subjected to mild to standard conditioning and a short 30 mins of antibody incubation at $37^{\circ} \mathrm{C}$. All sections were then treated with biotinylated secondary antibodies (Vector Laboratories, USA, RRIDs: AB_2336123, AB_2313606; Jackson ImmunoResearch Labs, RRID:AB_2338586), and later stained using the DAB method (Vectastain ABC-Peroxidase kits, Vector Laboratories, USA, RRID:AB_2336818). The sections were finally counterstained with hematoxylin.

\section{Immunofluorescence}

Paraffin-embedded human hippocampal sections were warmed on a hot-plate at approximately $45^{\circ} \mathrm{C}$ for $15-$ $20 \mathrm{~min}$, to melt the paraffin. Slides were then immediately transferred to fresh $100 \%$ xylene and processed through an ethanol hydration gradient (100, 90, 70, 50\% ethanol solutions for $5 \mathrm{~min}$ each), before immersion in distilled water. After deparaffinization, immunofluorescence was performed as previously described [48]. Briefly, sections were washed thrice in phosphate buffer saline (PBS), boiled in $10 \mathrm{mM}$ sodium citrate solution for antigen retrieval, blocked in 5\% serum in PBS with $0.1 \%$ Triton X-100 (0.1\% PBX solution) and then incubated overnight at $4{ }^{\circ} \mathrm{C}$ with primary antibodies. Sections were then washed thrice in $0.1 \%$ PBX solution, incubated with appropriate species-specific secondary antibodies conjugated with Alexa 488, 568 or 647 fluorophores (Invitrogen, USA; RRIDs: AB 2534088, $\mathrm{AB} \_$10563566, $\mathrm{AB} \_141778$ ) for $2 \mathrm{~h}$ at room temperature and then counterstained with DAPI (4',6-Diamidino-2Phenylindole, Dihydrochloride; Invitrogen, RRID:AB
2629482) to visualize nuclei. Sections were cover-slipped using Fluorogel mounting medium (Electron Microscopy Sciences, USA, Cat\# 50-247-04). Primary antibodies used: rabbit anti-Calbindin (Swant, Switzerland, RRID: AB_2721225), rat anti-CTIP2 (Abcam, UK, RRID:AB_ 2064130), rabbit anti-PAX6 (Biolegend, USA, RRID:AB 2565003), rat anti-TBR2 (anti-EOMES, eBioscience, USA, RRID:AB_11042577), rabbit anti-BLBP (Abcam, UK; RRID:AB_2100476), rabbit anti-IBA1 (FUJIFILM Wako Chemicals, USA; RRID:AB_839504). Immunostained sections were imaged in Olympus VS-120 slidescanner microscope using Olympus VS-Desktop 2.9 software, and later processed in Image $1.51 \mathrm{j} 8$ and ImageJ2 (NIH, Bethesda, Maryland, USA) and Olympus VSOlyvia 2.9 software programs respectively.

Each antibody was validated for immunofluorescence/ immunohistochemistry by the correspondent manufacturer, and these data are available publicly on the company websites with indicated catalog numbers. This was also validated by us in our experiments, replicating published/expected expression in appropriate control tissue. No outliers were encountered.

\section{Quantitative analysis}

Data was collected from H\&E-stained coronal sections. Thickness and length measurements of human hippocampal GC layer were made using Olympus VS-Olyvia 2.9, Olympus VS-Desktop 2.9 (Olympus Corporation, Tokyo, Japan) and ImageJ2 software programs (NIH, Bethesda, Maryland, USA). Maximum GC layer thickness in each GCD sample was measured at the region where the layer was the thickest. For the ratio measurement of bilaminar GC layers, the entire thickness of the DG was measured - from the edge proximal to the hilus to the edge proximal to the molecular layer. The individual thicknesses of the inner and outer layers were obtained as well. To correlate maximum GC layer thickness versus age, cadaveric sections showing compact, DA and TT phenotypes were compared with corresponding corrected age of death. To plot GCD length as a proportion of total DG length, the inner aspect of the DG was measured for 12 controls and 12 seizure cases, using one coronal hippocampal section with GCD per sample. Statistical significance was assessed using Welchcorrected t-test (for GCD length/Total length plot) and two-way ANOVA followed by Tukey post-hoc test (for GCD proportion plots across groups, age and PMI, GC layer thickness comparison plots, GCD occurrence with clinical diagnoses). Linear regression was used for the maximum GCD thickness versus age of death plots. These analyses were performed in GraphPad Prism v7.0 (GraphPad Software Inc., San Diego, USA) and in Microsoft Excel. Differences were considered significant at $p<0.05$. Data are represented as stacked and regular 
bar graphs and scatter plots with mean \pm SEM in Fig. 2 and Supplementary Fig. 2 (Additional File 1). Measurements for Supplementary Fig. 6 were obtained with Nikon Elements BR v3-2 (Nikon Corporation, Tokyo, Japan) and final data are represented as mean \pm SD.

\section{Results}

Defining types of GCD in seizure-affected human hippocampi

Stereotypically, the human hippocampal GC layer appears to have a compact, neuron-dense histology, with a sharp boundary separating the molecular layer (Fig. 1b', c,c'). While studying the cadaveric hippocampal samples from patients with history of epilepsy or seizure, we observed co-existence of compact GC layer and a range of GCD subtypes in the DG (Fig. 1d-d", e), as originally reported in hippocampal samples of TLE patients [30]. These were later classified by Blumcke et al. into different categories of DG granule cell pathology (GCP) [8]. This classification offered 3 major categories -1 ) entire DG appears normal, or non-GCP; 2) Type 1 GCP, characterized by severe cell loss; and 3) Type 2 GCP, that includes at least one DG focus of broadening, clustering or duplication of GC layer [8]. In our study, we further subdivided Type 2 GCP category broadly into two subtypes. The first is marked by broad, less dense GC layer, often with poorly defined borders with the molecular layer; we refer to this as "disaggregated" GCD (DA). The second subtype has a bilaminar appearance of the GC layer with cell-sparse zone in the center [3,30]; we refer to this as "tram-track" GCD (TT). By studying the H\&E-stained hippocampal specimens from patients with seizure history $(n=21)$, we categorized the morphology of GC layer for each as compact $(10 / 21 ; 47.62 \%$, subtype equivalent to non-GCP of [8]), only DA $(5 / 21 ; 23.81 \%)$, only TT (1/ $21 ; 4.76 \%)$, or both DA and TT $(5 / 21 ; 23.81 \%)$ (Table 1$)$. Focal granule cell loss was observed in only 1 seizure case (rigorous morphometric analysis of neuronal density was not performed). As previously reported [30], we also encountered dispersion and variation in the pattern and thickness of the GC layer at the angles and enfolded regions of both control and seizure-affected hippocampi. However, these areas were excluded from our consideration of "GCD" and also from our quantitative analyses.

\section{GCD is evident in brains irrespective of the history of seizures}

While evaluating age-matched cadaveric controls for seizure-affected brain specimens, we serendipitously identified GCD in some samples. This led us to retrospectively investigate the presence of GCD in a large set of 126 control human brains, with no history of seizures, and compare the findings with those observed in the 21 seizure cases (Additional File 2). We identified both DA

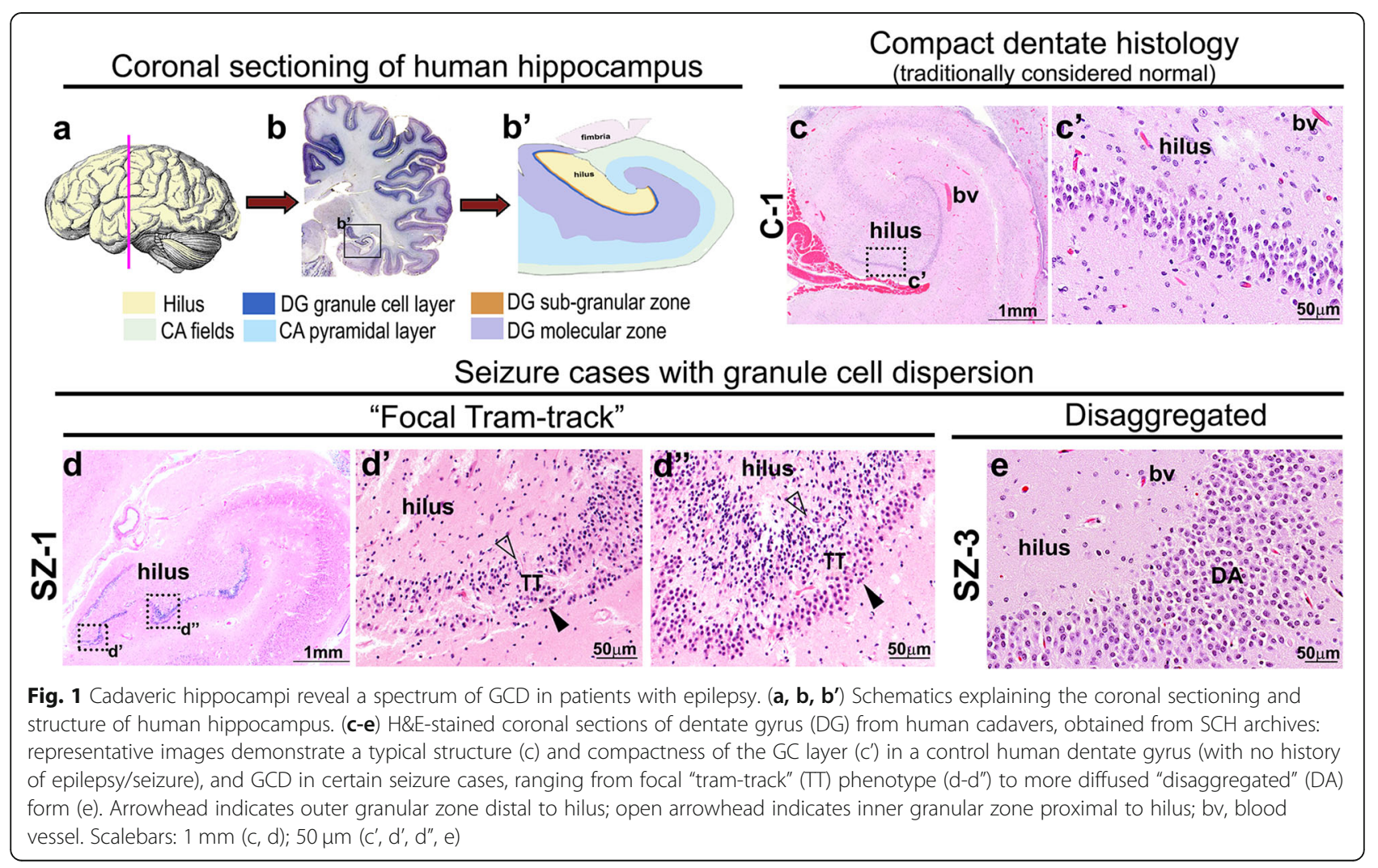


Table 1 List of cadaveric epilepsy cases with recorded clinical features. 21 cadaveric epilepsy cases with history of single or multiple events of seizures, accompanied with a table of clinical history obtained from the archives of Seattle Children's Hospital Department of Pathology (2014-2019), are tabulated. The features analyzed included age of death, gender, post-mortem interval (PMI), presence or absence of GCD subtypes, clinical diagnoses like seizure interval (from seizure onset until death), evidence of malformation/ anomaly in the central nervous system (CNS), and/or of other CNS (acquired forms of CNS injury such as hypoxic-ischemic encephalopathy (HIE), cerebral edema) and non-CNS conditions. GW, gestational weeks; TT, tram-track; DA, disaggregated; mo, months

\begin{tabular}{|c|c|c|c|c|c|c|c|c|c|c|c|}
\hline \multirow[t]{2}{*}{ Code } & \multirow{2}{*}{$\begin{array}{l}\text { Age of } \\
\text { death } \\
(G W)\end{array}$} & \multirow{2}{*}{$\begin{array}{l}\text { Corrected } \\
\text { age of } \\
\text { death }\end{array}$} & \multirow[t]{2}{*}{ Gender } & \multirow[t]{2}{*}{ PMI } & \multirow[t]{2}{*}{$\begin{array}{l}\text { Seizure } \\
(\mathrm{Y} / \mathrm{N})\end{array}$} & \multirow[t]{2}{*}{$\begin{array}{l}\text { Seizure } \\
\text { interval }\end{array}$} & \multicolumn{2}{|c|}{$\begin{array}{l}\text { GCD } \\
(\mathrm{Y} / \mathrm{N})\end{array}$} & \multicolumn{3}{|l|}{ Diagnoses } \\
\hline & & & & & & & $\overline{\pi T}$ & $\overline{D A}$ & $\begin{array}{l}\text { CNS malformation/ } \\
\text { anomaly }\end{array}$ & Other (CNS) & Non-CNS conditions \\
\hline SZ-1 ${ }^{a}$ & 7 days & 7 days & $M$ & $12 \mathrm{~h}$ & $Y$ & $<24 h$ & $Y$ & $Y$ & $\begin{array}{l}\text { encephalopathy, } \\
\text { seizures }\end{array}$ & - & $\begin{array}{l}\text { Proteus mirabilis septic } \\
\text { shock, coagulopathy } \\
\text { and acidosis, hypoxic } \\
\text { respiratory failure }\end{array}$ \\
\hline$S Z-2^{a}$ & 6 years & 6 years & $\mathrm{F}$ & $10 \mathrm{~h}$ & Y & $\begin{array}{l}6 \text { years; } \\
\text { intractable }\end{array}$ & $\mathrm{N}$ & $\mathrm{N}$ & $\begin{array}{l}\text { global developmental } \\
\text { delay, chronic seizure } \\
\text { disorder with static } \\
\text { encephalopathy }\end{array}$ & $\begin{array}{l}\text { hypoplastic } \\
\text { cerebellum }\end{array}$ & $\begin{array}{l}\text { recurrent pulmonary } \\
\text { infections }\end{array}$ \\
\hline$S Z-3^{a}$ & 16 years & 16 years & $\mathrm{F}$ & $22 \mathrm{~h}$ & Y & 13 years & $\mathrm{N}$ & Y & $\begin{array}{l}\text { severe congenital } \\
\text { neuromuscular } \\
\text { disorder, complex } \\
\text { status epilepticus }\end{array}$ & $\begin{array}{l}\text { severe gliosis } \\
\text { and neuronal } \\
\text { loss }\end{array}$ & $\begin{array}{l}\text { acute } \\
\text { bronchopneumonia, } \\
\text { cardiac arrest }\end{array}$ \\
\hline$S Z-4^{b}$ & 6 years & 6 years & M & $\begin{array}{l}\text { not } \\
\text { known }\end{array}$ & Y & $\begin{array}{l}\text { > } 5 \text { years; multiple } \\
\text { episodes till } \\
\text { death, intractable }\end{array}$ & Y & Y & $\begin{array}{l}\text { brain overgrowth } \\
\text { with polymicrogyria, } \\
\text { diffused cortical } \\
\text { dysplasia, AKT3 } \\
\text { R465W mutation, } \\
\text { possibly SUDEP }\end{array}$ & $\begin{array}{l}\text { gliosis, mild } \\
\text { ventriculomegaly }\end{array}$ & $\begin{array}{l}\text { congenital } \\
\text { diaphragmatic hernia, } \\
\text { coagulopathy, defects } \\
\text { in liver, spleen and } \\
\text { kidney }\end{array}$ \\
\hline$S Z-5^{a}$ & 3 years & 3 years & M & $2 \mathrm{~h}$ & Y & $\begin{array}{l}7 \text { weeks; multiple } \\
\text { episodes till } \\
\text { death, intractable }\end{array}$ & Y & $\mathrm{N}$ & $\begin{array}{l}\text { subclinical status } \\
\text { epilepticus }\end{array}$ & $\begin{array}{l}\text { cerebral edema, } \\
\text { HIE, anoxic brain } \\
\text { injury secondary } \\
\text { to pulmonary } \\
\text { arrest }\end{array}$ & pulmonary arrest \\
\hline$S Z-6^{a}$ & 2 days & 2 days & $\mathrm{F}$ & $21.5 \mathrm{~h}$ & Y & $\begin{array}{l}2 \text { days (possible } \\
\text { seizures) }\end{array}$ & Y & Y & - & $\begin{array}{l}\text { cerebral edema, } \\
\text { HIE }\end{array}$ & $\begin{array}{l}\text { acute chorioamnionitis } \\
\text { with funisitis and } \\
\text { three vessel umbilical } \\
\text { vasculitis }\end{array}$ \\
\hline $\mathrm{SZ}-\mathrm{7}^{\mathrm{a}}$ & 4 days & 4 days & M & $38 \mathrm{~h}$ & Y & $<24 h$ & $\mathrm{~N}$ & Y & - & HIE & $\begin{array}{l}\text { severe acidosis, } \\
\text { cardiorespiratory } \\
\text { failure, visceral } \\
\text { anomalies }\end{array}$ \\
\hline $\mathrm{SZ}-8^{\mathrm{a}}$ & $18 \mathrm{mo}$ & $18 \mathrm{mo}$ & M & $26 \mathrm{~h}$ & Y & $\begin{array}{l}1-3 \text { months of } \\
\text { age; no further } \\
\text { seizures } \\
\text { post-treatment } \\
\text { of phenobarbital }\end{array}$ & Y & Y & $\begin{array}{l}\text { developmental delay } \\
\text { and seizure disorder } \\
\text { (2q21.1 duplication) }\end{array}$ & $\begin{array}{l}\text { focal neuronal } \\
\text { loss and gliosis } \\
\text { in hippocampus }\end{array}$ & $\begin{array}{l}\text { abdominal distension, } \\
\text { brady-cardiac arrest, } \\
\text { acute pan-lobar } \\
\text { pneumonia }\end{array}$ \\
\hline SZ-9 ${ }^{\mathrm{a}}$ & 2 years & 2 years & $M$ & $72 \mathrm{~h}$ & Y & $\begin{array}{l}4 \text { months; multiple } \\
\text { episodes between } \\
\text { first seizure and } \\
\text { death }\end{array}$ & $N$ & $\mathrm{~N}$ & $\begin{array}{l}\text { retinoblastoma with } \\
\text { diffuse } \\
\text { leptomeningeal } \\
\text { spread and direct } \\
\text { infiltration of brain } \\
\text { and spinal cord }\end{array}$ & - & $\begin{array}{l}\text { pulmonary edema, } \\
\text { congestive } \\
\text { hepatomegaly }\end{array}$ \\
\hline$S Z-10^{a}$ & $\begin{array}{l}5 \text { weeks } \\
(28 \mathrm{GW})\end{array}$ & $33 \mathrm{GW}$ & $\mathrm{F}$ & $17.5 \mathrm{~h}$ & Y & $<24 h$ & Y & Y & $\begin{array}{l}\text { multiple seizure } \\
\text { events }\end{array}$ & $\begin{array}{l}\text { hemorrhage and } \\
\text { necrosis } \\
\text { secondary to } \\
\text { dural venous } \\
\text { thrombosis. }\end{array}$ & $\begin{array}{l}\text { necrotizing } \\
\text { enterocolitis }\end{array}$ \\
\hline$S Z-11^{a}$ & 12 years & 12 years & $\mathrm{F}$ & $96 \mathrm{~h}$ & Y & $\begin{array}{l}16 \text { months; } \\
\text { intractable }\end{array}$ & $N$ & $N$ & - & $\begin{array}{l}\text { cerebral edema, } \\
\text { HIE and brain } \\
\text { death }\end{array}$ & appendicitis \\
\hline$S Z-12^{a}$ & 19 years & 19 years & $M$ & $216 \mathrm{~h}$ & Y & $\begin{array}{l}14 \text { years; } \\
\text { intractable }\end{array}$ & $\mathrm{N}$ & $\mathrm{N}$ & seizure disorder & - & $\begin{array}{l}\text { Trisomy } 16 \mathrm{p} / \text { monosomy } \\
9 \mathrm{p} \text {, respiratory distress } \\
\text { syndrome, Pseudomonas } \\
\text { pneumonia, cardiomegaly }\end{array}$ \\
\hline
\end{tabular}


Table 1 List of cadaveric epilepsy cases with recorded clinical features. 21 cadaveric epilepsy cases with history of single or multiple events of seizures, accompanied with a table of clinical history obtained from the archives of Seattle Children's Hospital Department of Pathology (2014-2019), are tabulated. The features analyzed included age of death, gender, post-mortem interval (PMI), presence or absence of GCD subtypes, clinical diagnoses like seizure interval (from seizure onset until death), evidence of malformation/ anomaly in the central nervous system (CNS), and/or of other CNS (acquired forms of CNS injury such as hypoxic-ischemic encephalopathy (HIE), cerebral edema) and non-CNS conditions. GW, gestational weeks; TT, tram-track; DA, disaggregated; mo, months (Continued)

\begin{tabular}{|c|c|c|c|c|c|c|c|c|c|c|c|}
\hline \multirow[t]{2}{*}{ Code } & \multirow{2}{*}{$\begin{array}{l}\text { Age of } \\
\text { death } \\
(\mathrm{GW})\end{array}$} & \multirow{2}{*}{$\begin{array}{l}\text { Corrected } \\
\text { age of } \\
\text { death }\end{array}$} & \multirow[t]{2}{*}{ Gender } & \multirow[t]{2}{*}{ PMI } & \multirow[t]{2}{*}{$\begin{array}{l}\text { Seizure } \\
(\mathrm{Y} / \mathrm{N})\end{array}$} & \multirow[t]{2}{*}{$\begin{array}{l}\text { Seizure } \\
\text { interval }\end{array}$} & \multicolumn{2}{|c|}{$\begin{array}{l}\text { GCD } \\
(\mathrm{Y} / \mathrm{N})\end{array}$} & \multicolumn{3}{|l|}{ Diagnoses } \\
\hline & & & & & & & $\overline{\pi T}$ & $\overline{D A}$ & $\begin{array}{l}\text { CNS malformation/ } \\
\text { anomaly }\end{array}$ & Other (CNS) & Non-CNS conditions \\
\hline $\mathrm{SZ}-13^{\mathrm{a}}$ & $\begin{array}{l}7 \text { weeks } \\
(324 / 7 \mathrm{GW})\end{array}$ & $\begin{array}{l}394 / 7 \\
\text { GW }\end{array}$ & $\mathrm{F}$ & $14 \mathrm{~h}$ & $Y$ & $\begin{array}{l}6 \text { weeks (possible } \\
\text { seizures) }\end{array}$ & $\mathrm{N}$ & $Y$ & $\begin{array}{l}\text { epilepsy (focal } \\
\text { apoptotic neurons } \\
\text { within DG) }\end{array}$ & - & $\begin{array}{l}\text { severe pulmonary } \\
\text { hypertension, } \\
\text { veno-occlusive disease }\end{array}$ \\
\hline$S Z-14^{a}$ & 8 years & 8 years & $\mathrm{F}$ & $65 \mathrm{~h}$ & Y & $\begin{array}{l}2 \text { years (sleep } \\
\text { EEG done) }\end{array}$ & $N$ & $\mathrm{~N}$ & $\begin{array}{l}\text { encephalomalacia, } \\
\text { hydrocephalus, } \\
\text { seizures, } \\
\text { developmental delay }\end{array}$ & - & $\begin{array}{l}\text { unbalanced } \\
\text { chromosomal } \\
\text { translocation, } \\
\text { congenital mitral valve } \\
\text { stenosis, heart failure }\end{array}$ \\
\hline $\mathrm{SZ}-15^{\mathrm{a}}$ & 7 weeks & 7 weeks & $\mathrm{F}$ & $3 \mathrm{~h}$ & Y & 6 weeks & $N$ & Y & $\begin{array}{l}\text { hypotonia and } \\
\text { episodic breathing } \\
\text { progressing to } \\
\text { seizures }\end{array}$ & $\begin{array}{l}\text { elevated CSF } \\
\text { and plasma } \\
\text { glycine levels }\end{array}$ & - \\
\hline$S Z-16^{a}$ & 17 years & 17 years & $\mathrm{F}$ & $96 \mathrm{~h}$ & Y & 5 days & $N$ & $\mathrm{~N}$ & $\begin{array}{l}\text { generalized tonic- } \\
\text { clonic seizure, brain } \\
\text { herniation }\end{array}$ & $\begin{array}{l}\text { acute } \\
\text { hemorrhage, } \\
\text { edema }\end{array}$ & $\begin{array}{l}\text { Type } 1 \text { diabetes, } \\
\text { oligoarticular juvenile } \\
\text { arthritis, celiac disease }\end{array}$ \\
\hline $\mathrm{SZ}-17^{\mathrm{a}}$ & 17 years & 17 years & M & $64 \mathrm{~h}$ & Y & $\begin{array}{l}\text { 17 years; } \\
\text { intractable }\end{array}$ & $\mathrm{N}$ & Y & $\begin{array}{l}\text { spastic quadriplegia, } \\
\text { epilepsy, static } \\
\text { leukoencephalopathy, } \\
\text { ventriculomegaly }\end{array}$ & $\begin{array}{l}\text { white matter } \\
\text { gliosis }\end{array}$ & $\begin{array}{l}\text { acute kidney injury, } \\
\text { obstructive apnea, } \\
\text { hypotonia }\end{array}$ \\
\hline$S Z-18^{a}$ & 8 days & 8 days & M & $42 \mathrm{~h}$ & Y & $\begin{array}{l}8 \text { days } \\
\text { (onset at birth) }\end{array}$ & $\mathrm{N}$ & $\mathrm{N}$ & $\begin{array}{l}\text { seizures (treated with } \\
\text { antiepileptics) }\end{array}$ & $\begin{array}{l}\text { brain herniation, } \\
\text { diffuse cerebral } \\
\text { edema }\end{array}$ & $\begin{array}{l}\text { ornithine } \\
\text { transcarbamylase } \\
\text { deficiency, } \\
\text { hyperammonemia, } \\
\text { hepatosplenomegaly }\end{array}$ \\
\hline$S Z-19^{a}$ & 3 years & 3 years & $\mathrm{F}$ & $15.6 \mathrm{~h}$ & Y & 2.5 years & $N$ & $N$ & $\begin{array}{l}\text { severe craniofacial } \\
\text { malformations, seizure } \\
\text { history }\end{array}$ & $\begin{array}{l}\text { neurological } \\
\text { injury, meningitis }\end{array}$ & - \\
\hline$S Z-20^{a}$ & 4 years & 4 years & $M$ & $15.5 \mathrm{~h}$ & Y & 16 months & $\mathrm{N}$ & $\mathrm{N}$ & $\begin{array}{l}\text { seizures (no } \\
\text { recurrence post- } \\
\text { treatment with medi- } \\
\text { cations), global devel- } \\
\text { opmental delay }\end{array}$ & $\begin{array}{l}\text { subacute diffuse } \\
\text { CNS } \\
\text { hemorrhagic } \\
\text { necrosis with } \\
\text { massive } \\
\text { intraventricular } \\
\text { blood clot }\end{array}$ & $\begin{array}{l}\text { multiple chromosomal } \\
\text { abnormalities and } \\
\text { associated chronic } \\
\text { health problems, } \\
\text { atypical lymphoid } \\
\text { hyperplasia, concerning } \\
\text { primary or secondary } \\
\text { immunodeficiency }\end{array}$ \\
\hline$\underset{\mathrm{a}}{\mathrm{SZ}-21}$ & 9 years & 9 years & M & $2 \mathrm{~h}$ & Y & 2 months & $\mathrm{N}$ & $N$ & $\begin{array}{l}\text { refractory status } \\
\text { epilepticus secondary } \\
\text { to febrile infection- } \\
\text { related status epilepti- } \\
\text { cus, multiple events }\end{array}$ & $\begin{array}{l}\text { diffuse severe } \\
\text { gliosis, patchy } \\
\text { neuronal loss, } \\
\text { dramatic loss of } \\
\text { CA1 neurons }\end{array}$ & - \\
\hline
\end{tabular}

\footnotetext{
a based on microscopic evaluation of archived hippocampal sections

${ }^{b}$ case published in $[3,46]$; unused right hemisphere was obtained from SCH morgue and pathological studies were done by RPK on the right hippocampus for the first time for this study
}

and TT subtypes of GCD in control DG, similar to that seen in cadaveric epileptic brains (Fig. 2a-d', Table 2; Supplementary Fig. 1, Additional File 1). The proportion of each subtype observed in controls was as follows: compact $(76 / 126 ; 60.32 \%)$, only DA (27/126; $21.43 \%)$, only TT (2/126; $1.58 \%)$, both DA and TT (21/126; $16.67 \%)$. Statistical analysis between control and seizure cases, using two-way ANOVA, followed by Tukey post- hoc test, confirmed no significant difference across these 4 groups ( $p>0.9996$; Fig. 2e). Furthermore, we often observed the existence of more than one morphological type of GC layer (compact, DA, TT) in the same hippocampal section, irrespective of the seizure history, consistent with previous reports $[8,21]$. 


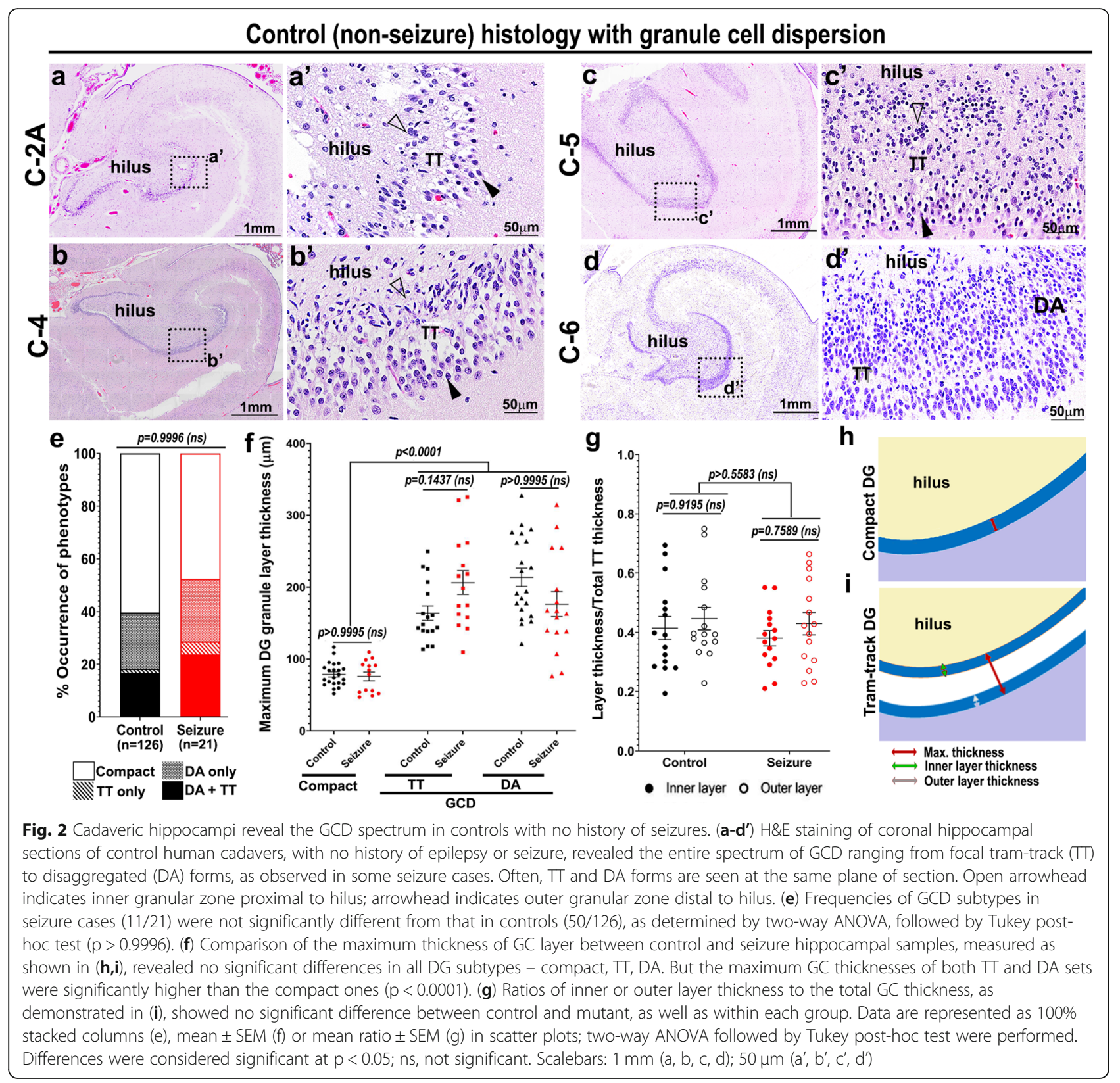

\section{Morphometric measurements of GCD are similar in seizure patients and controls}

Measuring the maximum thickness of compact, TT and DA sub-zones of GC layer demonstrated that both types of dispersed DG were significantly thicker than compact DG $(p<0.0001)$ in control and seizure-affected brains; however, no significant difference was observed between control and seizure cases in thicknesses of either the compact or dispersed areas (Fig. 2f,h,i). Moreover, for foci of tram-track GCD, the ratios of the inner or outer layer thicknesses to the total (maximum) GC layer thickness were not significantly different for both control and seizure-affected brains (Fig. 2g,i). Cell migration to the
GC layer occurs during the first 8 postnatal months, when immature cells gradually disappear from the subgranular zone near the hilus $[49,50]$. We categorized the age of death into bins and calculated the proportion of control and seizure cases per bin that demonstrated GCD. Statistical analyses showed no significant correlation between GCD occurrence and the age of death, in either control or seizure groups ( $p>0.2$; Supplementary Fig. 2a, Additional File 1). The PMI for the entire cohort largely varied between $2 \mathrm{~h}$ and $336 \mathrm{~h}$. Comparison of GCD and non-GCD numbers across different PMIs showed no significant correlation between development of GCD and PMI in both control 
Table 2 List of cadaveric controls with recorded clinical features, demonstrating GCD. 50 controls with no history of seizures, that demonstrated presence of at least one type of GCD in the studied hippocampal section obtained from the archives of Seattle Children's Hospital Department of Pathology (2014-2019), are tabulated. Other related clinical features obtained/analyzed were age of death, gender, post-mortem interval (PMI), presence or absence of GCD subtypes, clinical diagnoses like evidence of malformation/anomaly in the central nervous system (CNS), and/or of other CNS (acquired forms of CNS injury such as hypoxicischemic encephalopathy (HIE), cerebral edema) and non-CNS conditions. GW, gestational weeks; T, tram-track; DA, disaggregated; mo, months

\begin{tabular}{|c|c|c|c|c|c|c|c|c|c|c|}
\hline \multirow[t]{2}{*}{ Code } & \multirow{2}{*}{$\begin{array}{l}\text { Age of } \\
\text { death } \\
\text { (GW) }\end{array}$} & \multirow{2}{*}{$\begin{array}{l}\text { Corrected } \\
\text { age of } \\
\text { death }\end{array}$} & \multirow[t]{2}{*}{ Gender } & \multirow[t]{2}{*}{ PMI } & \multirow[t]{2}{*}{$\begin{array}{l}\text { Seizure } \\
(\mathrm{Y} / \mathrm{N})\end{array}$} & \multicolumn{2}{|c|}{$\begin{array}{l}\text { GCD } \\
(Y / N)\end{array}$} & \multicolumn{3}{|l|}{ Diagnoses } \\
\hline & & & & & & $\pi$ & DA & $\begin{array}{l}\text { CNS malformation/ } \\
\text { anomaly }\end{array}$ & Other (CNS) & Non-CNS conditions \\
\hline $\mathrm{C}-1^{\mathrm{a}}$ & $\begin{array}{l}1 \text { day } \\
(37 \mathrm{GW})\end{array}$ & $37 G W$ & $\mathrm{~F}$ & $14 \mathrm{~h}$ & $\mathrm{~N}$ & $\mathrm{~N}$ & $\begin{array}{l}\text { Y } \\
\text { (focal DA) }\end{array}$ & $\begin{array}{l}\text { large occipital } \\
\text { encephalocele, } \\
\text { focal dysplasia }\end{array}$ & $\begin{array}{l}\text { neuronal } \\
\text { disorganization, HIE }\end{array}$ & $\begin{array}{l}\text { multiple congenital } \\
\text { abnormalities }\end{array}$ \\
\hline $\begin{array}{l}C-2 A, \\
C-2 B^{a}\end{array}$ & $\begin{array}{l}4 \text { weeks } \\
(27 G W)\end{array}$ & $31 \mathrm{GW}$ & M & $32 \mathrm{~h}$ & $\mathrm{~N}$ & Y & Y & - & - & $\begin{array}{l}\text { RH-isoimmunization } \\
\text { hydrops fetalis, liver } \\
\text { failure, respiratory } \\
\text { distress }\end{array}$ \\
\hline$C-3^{a}$ & $\begin{array}{l}3 \text { weeks } \\
(26 \mathrm{GW})\end{array}$ & 29GW & M & $23 \mathrm{~h}$ & N & Y & Y & - & $\begin{array}{l}\text { intraventricular } \\
\text { hemorrhage, } \\
\text { frontoparietal } \\
\text { periventricular } \\
\text { leukomalacia }\end{array}$ & $\begin{array}{l}\text { necrotizing enterocolitis } \\
\text { and pneumatosis, sepsis }\end{array}$ \\
\hline$C-4^{a}$ & 3 weeks & 3 weeks & $\mathrm{F}$ & $57.5 \mathrm{~h}$ & N & Y & Y & - & diffuse edema & $\begin{array}{l}\text { congenital } \\
\text { diaphragmatic hernia, } \\
\text { coagulopathy, defects } \\
\text { in liver, spleen and } \\
\text { kidney }\end{array}$ \\
\hline$C-5^{a}$ & $\begin{array}{l}7 \text { weeks } \\
(27 \mathrm{GW})\end{array}$ & $28 \mathrm{GW}$ & M & $12 \mathrm{~h}$ & N & Y & Y & - & $\begin{array}{l}\text { HIE with } \\
\text { periventricular } \\
\text { leukomalacia }\end{array}$ & $\begin{array}{l}\text { multi-organ hypoxic } \\
\text { ischemic injury }\end{array}$ \\
\hline$C-6^{a}$ & $\begin{array}{l}2.5 \mathrm{mo} \\
\text { (term) }\end{array}$ & 10 weeks & $\mathrm{F}$ & $12 \mathrm{~h}$ & N & Y & Y & - & cerebral atrophy & $\begin{array}{l}\text { immunodeficiency } \\
\text { disorder of undefined } \\
\text { etiology, massive } \\
\text { hepatomegaly, active } \\
\text { bronchopneumonia, } \\
\text { cardiomegaly }\end{array}$ \\
\hline$C-7^{a}$ & $\begin{array}{l}15 \text { days } \\
(28 \mathrm{GW})\end{array}$ & $30 \mathrm{GW}$ & $\mathrm{F}$ & $6 \mathrm{~h}$ & $\mathrm{~N}$ & Y & Y & - & - & $\begin{array}{l}\text { congenital heart disease, } \\
\text { acute multifocal } \\
\text { pneumonia, congestion } \\
\text { and hemorrhage }\end{array}$ \\
\hline $\mathrm{C}-8^{\mathrm{a}}$ & $\begin{array}{l}3 \mathrm{mo} \\
(35 \mathrm{GW})\end{array}$ & 7 weeks & $\mathrm{F}$ & $17 \mathrm{~h}$ & N & Y & Y & - & $\begin{array}{l}\text { cerebral atrophy with } \\
\text { HIE, edema }\end{array}$ & $\begin{array}{l}\text { Trisomy 21, severe } \\
\text { hepatic fibrosis with } \\
\text { cholestasis, pneumonia, } \\
\text { cardiac defects, liver } \\
\text { and kidney injury }\end{array}$ \\
\hline$C-9^{a}$ & $2 \mathrm{mo}$ & 8 weeks & M & $20 \mathrm{~h}$ & N & Y & Y & - & $\begin{array}{l}\text { remote HIE without } \\
\text { acute changes, focal } \\
\text { cystic periventricular } \\
\text { leukomalacia }\end{array}$ & $\begin{array}{l}\text { neonatal gastroschisis } \\
\text { repair, cardiovascular } \\
\text { defects }\end{array}$ \\
\hline $\mathrm{C}-10^{\mathrm{a}}$ & $3 \mathrm{mo}$ & 12 weeks & $\mathrm{F}$ & $16 \mathrm{~h}$ & N & Y & Y & - & $\begin{array}{l}\text { subacute HIE with } \\
\text { uncal herniation }\end{array}$ & $\begin{array}{l}\text { congenital cardiovascular } \\
\text { defects, 15q26-qter } \\
\text { deletion, multi-organ } \\
\text { hypoxia }\end{array}$ \\
\hline $\mathrm{C}-11^{\mathrm{a}}$ & $\begin{array}{l}7 \text { days } \\
(32 \mathrm{GW})\end{array}$ & $33 \mathrm{GW}$ & $\mathrm{F}$ & $15 \mathrm{~h}$ & N & $Y$ & $Y$ & - & $\begin{array}{l}\text { subdural hematoma, } \\
\text { diffuse HIE with } \\
\text { widespread gliosis and } \\
\text { early mineralization, } \\
\text { periventricular } \\
\text { leukomalacia, } \\
\text { hemorrhage, few } \\
\text { pyknotic and } \\
\text { karyorrhectic cells noted } \\
\text { in hippocampus }\end{array}$ & $\begin{array}{l}\text { cystic necrosis of liver, } \\
\text { respiratory failure, } \\
\text { congested spleen }\end{array}$ \\
\hline
\end{tabular}


Table 2 List of cadaveric controls with recorded clinical features, demonstrating GCD. 50 controls with no history of seizures, that demonstrated presence of at least one type of GCD in the studied hippocampal section obtained from the archives of Seattle Children's Hospital Department of Pathology (2014-2019), are tabulated. Other related clinical features obtained/analyzed were age of death, gender, post-mortem interval (PMI), presence or absence of GCD subtypes, clinical diagnoses like evidence of malformation/anomaly in the central nervous system (CNS), and/or of other CNS (acquired forms of CNS injury such as hypoxicischemic encephalopathy (HIE), cerebral edema) and non-CNS conditions. GW, gestational weeks; T, tram-track; DA, disaggregated; mo, months (Continued)

\begin{tabular}{|c|c|c|c|c|c|c|c|c|c|c|}
\hline \multirow[t]{2}{*}{ Code } & \multirow{2}{*}{$\begin{array}{l}\text { Age of } \\
\text { death } \\
(G W)\end{array}$} & \multirow{2}{*}{$\begin{array}{l}\text { Corrected } \\
\text { age of } \\
\text { death }\end{array}$} & \multirow[t]{2}{*}{ Gender } & \multirow[t]{2}{*}{ PMI } & \multirow[t]{2}{*}{$\begin{array}{l}\text { Seizure } \\
(\mathrm{Y} / \mathrm{N})\end{array}$} & \multicolumn{2}{|c|}{$\begin{array}{l}\text { GCD } \\
(Y / N)\end{array}$} & \multicolumn{3}{|l|}{ Diagnoses } \\
\hline & & & & & & $\overline{T T}$ & $\mathrm{DA}$ & $\begin{array}{l}\text { CNS malformation/ } \\
\text { anomaly }\end{array}$ & Other (CNS) & Non-CNS conditions \\
\hline $\mathrm{C}-12^{\mathrm{a}}$ & $\begin{array}{l}2 \mathrm{mo} \\
(37 \mathrm{GW})\end{array}$ & 5 weeks & $\mathrm{F}$ & $8 \mathrm{~h}$ & $\mathrm{~N}$ & Y & Y & - & $\begin{array}{l}\text { diffuse mild } \\
\text { cerebral WM gliosis }\end{array}$ & $\begin{array}{l}\text { complex congenital } \\
\text { heart disease, } \\
\text { cardiomegaly, aspiration } \\
\text { pneumonitis }\end{array}$ \\
\hline$C-13^{a}$ & 8 years & 8 years & $\mathrm{F}$ & $7 \mathrm{~h}$ & $\mathrm{~N}$ & $N$ & Y & - & $\begin{array}{l}\text { hemorrhagic } \\
\text { infarction, mild WM } \\
\text { atrophy, DG } \\
\text { hypoplasia and } \\
\text { neuronal loss, gliosis }\end{array}$ & $\begin{array}{l}\text { methylmalonic acidemia, } \\
\text { chronic liver failure, } \\
\text { coagulopathy, severe } \\
\text { diffuse } \\
\text { bronchopneumonia, } \\
\text { recurrent fevers }\end{array}$ \\
\hline$C-14^{a}$ & $\begin{array}{l}2 \mathrm{mo} \\
\text { (term) }\end{array}$ & 8 weeks & $\mathrm{F}$ & $20 \mathrm{~h}$ & $\mathrm{~N}$ & Y & Y & - & $\begin{array}{l}\text { remote HIE without } \\
\text { acute changes, focal } \\
\text { cystic periventricular } \\
\text { leukomalacia }\end{array}$ & $\begin{array}{l}\text { neonatal gastroschisis } \\
\text { repair, cardiovascular } \\
\text { defects, Clostridium } \\
\text { infection }\end{array}$ \\
\hline$C-15^{a}$ & 18 days & 18 days & M & $13.5 \mathrm{~h}$ & $\mathrm{~N}$ & Y & Y & - & $\begin{array}{l}\text { kernicterus involving } \\
\text { hippocampi, diffuse } \\
\text { gliosis with } \\
\text { periventricular } \\
\text { eukomalacia }\end{array}$ & $\begin{array}{l}\text { Beckwith-Wiedemann } \\
\text { Syndrome, respiratory } \\
\text { failure, acute kidney } \\
\text { injury, thymic cortical } \\
\text { stress }\end{array}$ \\
\hline$C-16^{a}$ & $\begin{array}{l}10 \text { weeks } \\
(32 \mathrm{GW})\end{array}$ & 2 weeks & M & $11 \mathrm{~h}$ & $\mathrm{~N}$ & $N$ & Y & - & - & $\begin{array}{l}\text { liver dysfunction of } \\
\text { uncertain etiology, } \\
\text { cytomegalovirus } \\
\text { infection }\end{array}$ \\
\hline$C-17^{a}$ & $\begin{array}{l}23 \text { days } \\
(25 G W)\end{array}$ & 28GW & M & $16.25 \mathrm{~h}$ & $\mathrm{~N}$ & Y & Y & - & $\begin{array}{l}\text { severe intracranial } \\
\text { hemorrhage }\end{array}$ & $\begin{array}{l}\text { necrotizing enterocolitis, } \\
\text { severe pneumonia, } \\
\text { pulmonary hemorrhage }\end{array}$ \\
\hline$C-18^{a}$ & 6 years & 6 years & $\mathrm{F}$ & $68 \mathrm{~h}$ & $\mathrm{~N}$ & Y & Y & - & $\begin{array}{l}\text { craniosynostosis } \\
\text { surgery }\end{array}$ & $\begin{array}{l}\text { GLIS3 mutation, hepatic } \\
\text { fibrosis }\end{array}$ \\
\hline$C-19^{a}$ & 6 years & 6 years & M & $15 \mathrm{~h}$ & $\mathrm{~N}$ & Y & Y & $\begin{array}{l}\text { diffuse infiltrating } \\
\text { pontine glioma, } \\
\text { mild ventriculomegaly }\end{array}$ & - & - \\
\hline $\begin{array}{l}C- \\
20^{b}\end{array}$ & $\begin{array}{l}12 \mathrm{~h} \\
(38 \mathrm{GW})\end{array}$ & 38GW & M & $38.5 \mathrm{~h}$ & $\mathrm{~N}$ & Y & Y & - & HIE & $\begin{array}{l}\text { asystole at birth, } \\
\text { bilaterallydilated ureters } \\
\text { and bladder, increased } \\
\text { extramedullary } \\
\text { hematopoiesis }\end{array}$ \\
\hline $\mathrm{C}-21^{\mathrm{a}}$ & $\begin{array}{l}3 \text { days } \\
(38 \mathrm{GW})\end{array}$ & $38 \mathrm{GW}$ & M & $15.75 \mathrm{~h}$ & $N$ & $N$ & Y & - & mild HIE and edema & $\begin{array}{l}\text { hemorrhagic and } \\
\text { necrotic small bowel, } \\
\text { anomalies in alimentary } \\
\text { tract, liver failure }\end{array}$ \\
\hline $\mathrm{C}-22^{\mathrm{a}}$ & $\begin{array}{l}8 \text { weeks } \\
(34 \mathrm{GW})\end{array}$ & 2 weeks & M & $8 \mathrm{~h}$ & $\mathrm{~N}$ & Y & Y & $\begin{array}{l}\text { mild } \\
\text { ventriculomegaly }\end{array}$ & $\begin{array}{l}\text { mild diffuse gliosis of } \\
\text { white matter }\end{array}$ & $\begin{array}{l}\text { Pentalogy of Cantrell, } \\
\text { left pulmonary artery } \\
\text { stenosis }\end{array}$ \\
\hline$C-23^{a}$ & 10 years & 10 years & M & $11 \mathrm{~h}$ & $N$ & Y & Y & - & & $\begin{array}{l}\text { immunodeficiency, } \\
\text { Pseudomonas and } \\
\text { Aspergillus infection }\end{array}$ \\
\hline$C-24^{a}$ & $\begin{array}{l}3 \text { days } \\
(38 \mathrm{GW})\end{array}$ & 38GW & M & $15.75 \mathrm{~h}$ & $\mathrm{~N}$ & $N$ & Y & - & mild edema and HIE & $\begin{array}{l}\text { hemorrhagic and } \\
\text { necrotic small bowel, } \\
\text { anomalies in alimentary } \\
\text { tract, liver failure }\end{array}$ \\
\hline$C-25^{\mathrm{a}}$ & 17 years & 17 years & M & $59 \mathrm{~h}$ & $\mathrm{~N}$ & $N$ & Y & - & - & $\begin{array}{l}\text { recurrent B cell } \\
\text { lymphoblastic leukemia } \\
\text { and aspergillosis }\end{array}$ \\
\hline
\end{tabular}


Table 2 List of cadaveric controls with recorded clinical features, demonstrating GCD. 50 controls with no history of seizures, that demonstrated presence of at least one type of GCD in the studied hippocampal section obtained from the archives of Seattle Children's Hospital Department of Pathology (2014-2019), are tabulated. Other related clinical features obtained/analyzed were age of death, gender, post-mortem interval (PMI), presence or absence of GCD subtypes, clinical diagnoses like evidence of malformation/anomaly in the central nervous system (CNS), and/or of other CNS (acquired forms of CNS injury such as hypoxicischemic encephalopathy (HIE), cerebral edema) and non-CNS conditions. GW, gestational weeks; T, tram-track; DA, disaggregated; mo, months (Continued)

\begin{tabular}{|c|c|c|c|c|c|c|c|c|c|c|}
\hline \multirow[t]{2}{*}{ Code } & \multirow{2}{*}{$\begin{array}{l}\text { Age of } \\
\text { death } \\
(G W)\end{array}$} & \multirow{2}{*}{$\begin{array}{l}\text { Corrected } \\
\text { age of } \\
\text { death }\end{array}$} & \multirow[t]{2}{*}{ Gender } & \multirow[t]{2}{*}{ PMI } & \multirow[t]{2}{*}{$\begin{array}{l}\text { Seizure } \\
(\mathrm{Y} / \mathrm{N})\end{array}$} & \multicolumn{2}{|c|}{$\begin{array}{l}\mathrm{GCD} \\
(\mathrm{Y} / \mathrm{N})\end{array}$} & \multicolumn{3}{|l|}{ Diagnoses } \\
\hline & & & & & & $\overline{T T}$ & $\mathrm{DA}$ & $\begin{array}{l}\text { CNS malformation/ } \\
\text { anomaly }\end{array}$ & Other (CNS) & Non-CNS conditions \\
\hline $\mathrm{C}-26^{\mathrm{a}}$ & $4 \mathrm{mo}$ & $4 \mathrm{mo}$ & M & $55 \mathrm{~h}$ & $\mathrm{~N}$ & $\mathrm{~N}$ & $Y$ & $\begin{array}{l}\text { axonal mixed } \\
\text { sensory/ motor } \\
\text { neuropathy }\end{array}$ & deafness & $\begin{array}{l}\text { growth delay, respiratory } \\
\text { distress }\end{array}$ \\
\hline $\mathrm{C}-27^{\mathrm{a}}$ & $\begin{array}{l}2 \mathrm{mo} \\
(32 \mathrm{GW})\end{array}$ & $\begin{array}{l}\text { term } \\
(40 G W)\end{array}$ & $\mathrm{F}$ & $58 \mathrm{~h}$ & $\mathrm{~N}$ & $N$ & Y & - & $\begin{array}{l}\text { periventricular } \\
\text { leukomalacia with } \\
\text { acute HIE }\end{array}$ & $\begin{array}{l}\text { cardiopulmonary } \\
\text { abnormalities, congenital } \\
\text { cardiac anomalies, } \\
\text { renomegaly }\end{array}$ \\
\hline$C-28^{a}$ & 1 week & 1 week & M & $45 \mathrm{~h}$ & $\mathrm{~N}$ & $N$ & Y & - & $\begin{array}{l}\text { HIE, periventricular } \\
\text { leukomalacia with } \\
\text { prominent gliosis } \\
\text { and neuronal loss }\end{array}$ & $\begin{array}{l}\text { congestion and } \\
\text { hemorrhage }\end{array}$ \\
\hline$C-29^{a}$ & $10 \mathrm{mo}$ & $10 \mathrm{mo}$ & $\mathrm{F}$ & $46.5 \mathrm{~h}$ & $\mathrm{~N}$ & $N$ & Y & - & $\begin{array}{l}\text { global chronic HIE, } \\
\text { hippocampus shows } \\
\text { mild loss of neurons } \\
\text { in CA1 region }\end{array}$ & $\begin{array}{l}\text { heterotaxy syndrome, } \\
\text { complex congenital } \\
\text { heart disease }\end{array}$ \\
\hline $\mathrm{C}-30^{\mathrm{a}}$ & 3 years & 3 years & M & $16.5 \mathrm{~h}$ & $\mathrm{~N}$ & $N$ & Y & - & $\begin{array}{l}\text { HIE post cardiac arrest, } \\
\text { early necrosis of } \\
\text { hippocampus }\end{array}$ & $\begin{array}{l}\text { asthma, acute sepsis, } \\
\text { cardiac arrest, stress } \\
\text { atrophy }\end{array}$ \\
\hline $\mathrm{C}-31^{\mathrm{a}}$ & $\begin{array}{l}3 \mathrm{mo} \\
(42 \mathrm{GW})\end{array}$ & 101 days & $\mathrm{F}$ & $13 \mathrm{~h}$ & $\mathrm{~N}$ & $N$ & Y & - & mild HIE & $\begin{array}{l}\text { respiratory distress, } \\
\text { pulmonary vein stenosis }\end{array}$ \\
\hline$C-32^{a}$ & $\begin{array}{l}14 \text { days } \\
\text { (28 week) }\end{array}$ & $30 \mathrm{GW}$ & M & $16 \mathrm{~h}$ & $\mathrm{~N}$ & $N$ & Y & - & $\begin{array}{l}\text { mild HIE, diffuse gliosis } \\
\text { in WM }\end{array}$ & $\begin{array}{l}\text { massive subacute } \\
\text { hepatic necrosis with } \\
\text { iron overload, } \\
\text { coagulopathy, chronic } \\
\text { neonatal lung disease, } \\
\text { multiple organ defects }\end{array}$ \\
\hline$C-33^{a}$ & 3 years & 3 years & $M$ & $20.5 \mathrm{~h}$ & $\mathrm{~N}$ & $N$ & Y & $\begin{array}{l}\text { global developmental } \\
\text { delay }\end{array}$ & HIE with edema & $\begin{array}{l}\text { myopathy, cardiac } \\
\text { failure, respiratory } \\
\text { failure, infectious } \\
\text { diseases, respiratory } \\
\text { distress, sepsis }\end{array}$ \\
\hline$C-34^{a}$ & $\begin{array}{l}\text { 5weeks } \\
(33 G W)\end{array}$ & $39 \mathrm{GW}$ & $\mathrm{F}$ & $84 \mathrm{~h}$ & $\mathrm{~N}$ & $N$ & Y & - & - & necrotizing enterocolitis \\
\hline$C-35^{\mathrm{a}}$ & $18 \mathrm{~h}$ & $18 \mathrm{~h}$ & M & $14 \mathrm{~h}$ & $\mathrm{~N}$ & $N$ & Y & - & - & $\begin{array}{l}\text { complex congenital } \\
\text { heart disease, total } \\
\text { anomalous pulmonary } \\
\text { venous return, } \\
\text { lymphatic distention }\end{array}$ \\
\hline $\mathrm{C}-36^{\mathrm{a}}$ & 17 years & 17 years & $\mathrm{F}$ & $69.5 h$ & $\mathrm{~N}$ & Y & Y & $\begin{array}{l}\text { medulloblastoma, } \\
\text { brain injury related } \\
\text { to Aspergillus } \\
\text { encephalo-meningitis, } \\
\text { lateral ventriculomegaly }\end{array}$ & widespread brain death & $\begin{array}{l}\text { pulmonary thrombi } \\
\text { and congestion and } \\
\text { hepatosplenomegaly }\end{array}$ \\
\hline$C-37^{a}$ & 9 days & 9 days & $\mathrm{F}$ & $31 \mathrm{~h}$ & N & $N$ & Y & - & HIE, brain injury & liver steatosis \\
\hline$C-38^{a}$ & $\begin{array}{l}6 \mathrm{~h} \\
(415 / 7 \mathrm{GW})\end{array}$ & 1.5 weeks & $\mathrm{F}$ & $78 \mathrm{~h}$ & $\mathrm{~N}$ & $N$ & Y & - & HIE & $\begin{array}{l}\text { cardiac respiratory } \\
\text { failure, coagulopathy, } \\
\text { anemia, severe } \\
\text { metabolic acidosis, } \\
\text { in-utero feto-maternal } \\
\text { hemorrhage }\end{array}$ \\
\hline$C-39^{a}$ & $16 \mathrm{mo}$ & $16 \mathrm{mo}$ & M & $15 \mathrm{~h}$ & $N$ & $\mathrm{~N}$ & $Y$ & - & $\begin{array}{l}\text { multifocal brain } \\
\text { infarction with global } \\
\text { HIE (CA1 dispersed) }\end{array}$ & $\begin{array}{l}\text { diffuse adherent bowel, } \\
\text { necrotizing soft tissue } \\
\text { infections, cardiac } \\
\text { arrest history }\end{array}$ \\
\hline
\end{tabular}


Table 2 List of cadaveric controls with recorded clinical features, demonstrating GCD. 50 controls with no history of seizures, that demonstrated presence of at least one type of GCD in the studied hippocampal section obtained from the archives of Seattle Children's Hospital Department of Pathology (2014-2019), are tabulated. Other related clinical features obtained/analyzed were age of death, gender, post-mortem interval (PMI), presence or absence of GCD subtypes, clinical diagnoses like evidence of malformation/anomaly in the central nervous system (CNS), and/or of other CNS (acquired forms of CNS injury such as hypoxicischemic encephalopathy (HIE), cerebral edema) and non-CNS conditions. GW, gestational weeks; T, tram-track; DA, disaggregated; mo, months (Continued)

\begin{tabular}{|c|c|c|c|c|c|c|c|c|c|c|}
\hline \multirow[t]{2}{*}{ Code } & \multirow{2}{*}{$\begin{array}{l}\text { Age of } \\
\text { death } \\
(G W)\end{array}$} & \multirow{2}{*}{$\begin{array}{l}\text { Corrected } \\
\text { age of } \\
\text { death }\end{array}$} & \multirow[t]{2}{*}{ Gender } & \multirow[t]{2}{*}{ PMI } & \multirow[t]{2}{*}{$\begin{array}{l}\text { Seizure } \\
(\mathrm{Y} / \mathrm{N})\end{array}$} & \multicolumn{2}{|c|}{$\begin{array}{l}\text { GCD } \\
(Y / N)\end{array}$} & \multicolumn{3}{|l|}{ Diagnoses } \\
\hline & & & & & & $\Pi T$ & DA & $\begin{array}{l}\text { CNS malformation/ } \\
\text { anomaly }\end{array}$ & Other (CNS) & Non-CNS conditions \\
\hline $\mathrm{C}-40^{\mathrm{a}}$ & 4 weeks & 4 weeks & $\mathrm{F}$ & $24 \mathrm{~h}$ & $\mathrm{~N}$ & Y & Y & - & $\begin{array}{l}\text { mild HIE with mild } \\
\text { gliosis }\end{array}$ & truncus arteriosus \\
\hline $\mathrm{C}-41^{\mathrm{a}}$ & $\begin{array}{l}4 \text { days } \\
(27 G W)\end{array}$ & $27 \mathrm{GW}$ & $\mathrm{F}$ & $144 \mathrm{~h}$ & $\mathrm{~N}$ & $\mathrm{~N}$ & Y & - & widespread HIE & splenic congestion \\
\hline$C-42^{a}$ & 8 years & 8 years & $\mathrm{F}$ & $15 \mathrm{~h}$ & $\mathrm{~N}$ & $N$ & Y & - & subdural hematoma & $\begin{array}{l}\text { B-cell acute } \\
\text { lymphoblastic } \\
\text { leukemia, sepsis, } \\
\text { acute kidney injury, } \\
\text { cardiac instability }\end{array}$ \\
\hline$C-43^{a}$ & $6 \mathrm{mo}$ & $6 \mathrm{mo}$ & M & $41.5 \mathrm{~h}$ & $\mathrm{~N}$ & $\mathrm{~N}$ & Y & - & global remote HIE & $\begin{array}{l}\text { Denys-Drash Syndrome, } \\
\text { chronic kidney disease, } \\
\text { Pseudomonas abscess, } \\
\text { multiple cardiac arrests }\end{array}$ \\
\hline$C-44^{a}$ & $\begin{array}{l}6 \text { years } \\
6 \mathrm{mo}\end{array}$ & $\begin{array}{l}6 \text { years } \\
6 \mathrm{mo}\end{array}$ & $\mathrm{F}$ & $13 \mathrm{~h}$ & $\mathrm{~N}$ & $\mathrm{~N}$ & Y & $\begin{array}{l}\text { diffuse intrinsic } \\
\text { pontine glioma }\end{array}$ & - & - \\
\hline$C-45^{a}$ & $\begin{array}{l}3 \text { days } \\
(401 / 7 G W)\end{array}$ & $40 G W$ & $\mathrm{~F}$ & $40.5 \mathrm{~h}$ & $\mathrm{~N}$ & $\mathrm{~N}$ & Y & - & - & $\begin{array}{l}\text { profound hypoxemic } \\
\text { respiratory failure, } \\
\text { lung developmental } \\
\text { arrest }\end{array}$ \\
\hline$C-46^{a}$ & $\begin{array}{l}3 \text { weeks } \\
(35 \mathrm{GW})\end{array}$ & $38 \mathrm{GW}$ & M & $19 \mathrm{~h}$ & $\mathrm{~N}$ & $N$ & Y & - & acute HIE & $\begin{array}{l}\text { congenital heart } \\
\text { disease, kidney } \\
\text { hemorrhage }\end{array}$ \\
\hline$C-47^{a}$ & 16 days & 16 days & $M$ & $10 \mathrm{~h}$ & N & $\mathrm{N}$ & Y & - & $\begin{array}{l}\text { HIE, diffuse WM gliosis, } \\
\text { periventricular leukomalacia, } \\
\text { subarachnoid hemorrhage }\end{array}$ & $\begin{array}{l}\text { complex congenital } \\
\text { heart disease, status } \\
\text { post-surgical repair }\end{array}$ \\
\hline$C-48^{a}$ & 7 weeks & 7 weeks & M & $50 \mathrm{~h}$ & $\mathrm{~N}$ & $\mathrm{~N}$ & Y & - & - & necrotizing enterocolitis \\
\hline$C-49^{a}$ & $35 G W$ & $35 \mathrm{GW}$ & M & $63 \mathrm{~h}$ & $\mathrm{~N}$ & Y & N & - & - & $\begin{array}{l}\text { congenital pulmonary } \\
\text { dysplasia, interstitial } \\
\text { chromosomal deletion } \\
\text { ch17 }\end{array}$ \\
\hline$C-50^{a}$ & 6 days & 6 days & M & $69 h$ & N & Y & N & - & subicular necrosis, acute HIE & $\begin{array}{l}\text { 22q11.2 chromosomal } \\
\text { deletion, DiGeorge } \\
\text { syndrome }\end{array}$ \\
\hline
\end{tabular}

${ }^{a}$ based on microscopic evaluation of archived hippocampal sections

b step sections as described in text

Table 3 Summary of study parameters. Summary table of variables used in this retrospective study to compare between the control (no seizure history) and seizure groups. Variables shown are corrected age of death, post-mortem interval (PMI), gender and seizure interval (from seizure onset until death). Comparison demonstrated broad overlap especially in the range of age of death and PMI between control and seizure sets

\begin{tabular}{llllll}
\hline Group & Number of cases & Range of corrected age of death & Range of PMI & Gender (\% of total) & Range of seizure interval \\
\hline Control & 126 & -21 to +1060 weeks & 2 to $336 \mathrm{~h}$ & Male: $69(54.76 \%) ;$ Female: $57(45.24 \%)$ & - \\
Seizure & 21 & -7 to +990.7 weeks & 2 to $216 \mathrm{~h}$ & Male: $11(52.38 \%) ;$ Female: 10 (47.62\%) & $<24 \mathrm{~h}$ to 17 years \\
\hline
\end{tabular}


and seizure groups (Supplementary Fig. 2b, Additional File 1). Comparison of maximum GC layer thickness in compact, DA and TT forms showed that both disaggregated and "tram-track" GC layer thicknesses were wider than the "compact" thickness for both control and seizure brains, across ages of the cadaveric samples (Supplementary Fig. 2c, c', Additional File 1). While other groups showed no correlation with the age of death, the "tram-track"-ed seizure cases (Seizure TT) demonstrated a slightly positive correlation of GC layer thickness with increasing age beyond 60 weeks $\left(R^{2}=0.4517, p=0.0167\right.$; Supplementary Fig. 2c, Additional File 1). Table 3 summarizes the overlapping range of clinical parameters (age of death, PMI, gender proportion) between control and seizure groups used in this study.

Further, we segregated the patient history into different diagnostic categories and analyzed whether putative GCD occurrence is dependent on any particular clinical diagnoses (Supplementary Fig. 2d, Additional File 1). Two-way ANOVA revealed no statistically significant correlation between the different diagnostic subgroups of patients and the presence of GCD in either control or seizure cases. We observed higher incidence of diagnoses related to the central nervous system for seizure cases than controls; however due to relatively low sample size of the seizure cases, this was also not considered statistically significant. Finally, we calculated the proportion of GC layer affected by GCD per plane of section in controls and epileptic brains and found no significant differences between them ( $p=0.801$; Supplementary Fig. 2e, Additional File 1). Hence, GCD was found to occur at a similar proportion in both control and seizure-affected brains and this occurrence was largely independent of age, gender, PMI or clinical diagnoses.

\section{Molecular expression of cells is identical in both control and seizure-affected dentate gyri with GCD}

To investigate molecular differences between control and seizure-affected brains, we performed immunohistochemistry and immunofluorescence on the cadaveric hippocampal sections. PROX1, marking cells in the adult GC layer and sub-granular zone [34], was expressed similarly in both control and seizure-affected DG (Fig. 3a-c,m,n). CTIP2 is typically expressed in the hippocampal CA1 and post-mitotic dentate granule cells [47], predominantly in the superficial GC layer; its expression in human DG gets attenuated gradually after mid-gestation [12]. In normal human DG, Calbindin immunostaining is observed in hilus, molecular and GC layers, with strongest expression in the early-born postmitotic neurons, located in the outer part GC layer distal to the hilar region [2]. In human brains affected by different types of epilepsy and/or hippocampal sclerosis,
Calbindin is noted to be prominent in outer granule cells and absent or sparse in the inner layer/half of the GC layer; however no overt cell loss were observed in most of these brains [1]. Similarly in our study, Calbindin and CTIP2 expression was stronger in the outer layer of the compact as well as "tram-track" control and epileptic DG, compared to the inner GC layer (Fig. 3d,e, $\mathrm{g}, \mathrm{h}, \mathrm{o}, \mathrm{q})$. On the other hand, CTIP2 and Calbindin were expressed in the entire "disaggregated" GC layer, in both controls and seizure cases (Fig. 3f,i,p,r). BLBP was expressed in the hilus and the molecular layer of the dentate in both control and seizure-affected brains (Fig. 3j-l, s,t), as expected [38, 53].

To determine whether the inner layer of tram-track DG corresponds to the sub-granular zone harboring progenitors and immature neurons, we examined the distributions of putative neural progenitor markers (SOX2, PAX6, TBR2) in control and seizure-affected hippocampi (Supplementary Fig. 3a-o, Additional File 1) $[12,61]$. No overt differences were observed in the expression of these markers within the dispersed (DA or TT) dentate gyri of control or seizure hippocampi. Our data are thus consistent with the study that identified disassociation of proliferation with GCD in the brains of TLE patients [20].

\section{GCD is not dependent on increased gliosis or injury-} driven mechanisms irrespective of seizure occurrence To determine whether tissue injury or macrophagerelated inflammation is associated with the GCD phenotypes, we examined the hippocampal expression of injury markers GFAP and CD163 in 6 seizure patients and 10 controls (Fig. 4) [18, 19]. Enhancement of strongly expressing $\mathrm{GFAP}^{+}$and $\mathrm{CD} 163^{+}$ cells denotes activation of astrocytes (gliosis) and inflammation-related M2 macrophages respectively, both indicative of tissue injury $[19,57]$. We observed increased gliosis in some of the cadaveric seizureaffected hippocampi, independent of GCD (Fig. 4b,f, h), although the DG in every epilepsy case did not have increased $\mathrm{GFAP}^{+}$astrocytes (Fig. 4d). Enhanced GFAP expression or gliosis was generally not observed in the control sections, irrespective of presence of GCD (Fig. 4j,l,n,p); among the 10 controls evaluated immunohistochemically, we encountered only 2 control brains that exhibited mild gliosis (as represented in Fig. 4r). We also did not observe any significant differences in the number of $\mathrm{CD}_{163}{ }^{+}$cells in the studied cadaveric control or epileptic hippocampi (Fig. 4c,e,g,i,k,m,o,q,s). Further, we studied the expression of CD68 and IBA1, generic markers for macrophages and microglia respectively, which congregate in response to tissue injury and cellular activation $[28,57]$. Similar to $\mathrm{CD} 163, \mathrm{CD}^{+} 8^{+}$cells were 


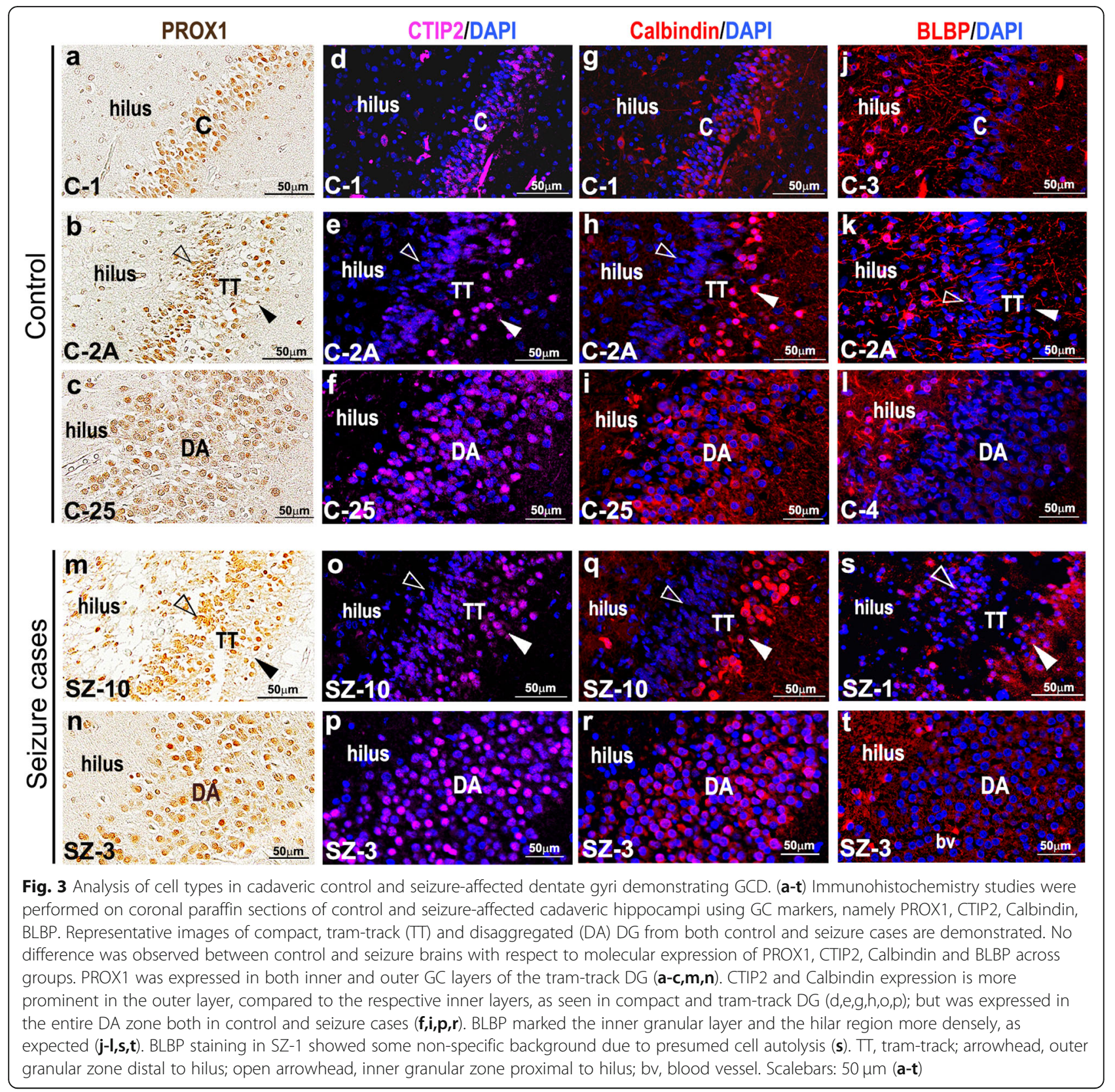

minimal and expressed non-differentially between control and epileptic brains (Supplementary Fig. 4ae, k-o; Additional File 1). We did encounter a few $\mathrm{IBA}^{+}$activated microglia, identified by rounded, bushy cellular morphology; but there was no correlation between their presence and GCD or seizure occurrence (Supplementary Fig. 4f-j, p-t; Additional File 1). Thus, our results indicate that occurrence of dispersed GC layer in both controls and seizure cases is not directly associated with either injury or inflammation, although gliosis appeared to be more common in epileptic hippocampi.
Extent of GCD changes across antero-posterior levels of human hippocampus

While analyzing multiple sections from the cadaveric hippocampal paraffin blocks, we observed that GCD was present inconsistently and to variable extents in different sectioning planes of both control and seizure-affected hippocampi (Supplementary Fig. 5ad", Additional File 1). This suggested that the putative seizure-related nature of "disaggregated" and/or "tram-track" GC layer in the literature might merely be a consequence of sampling bias. As mentioned in the Methods, our designation of controls and seizure 


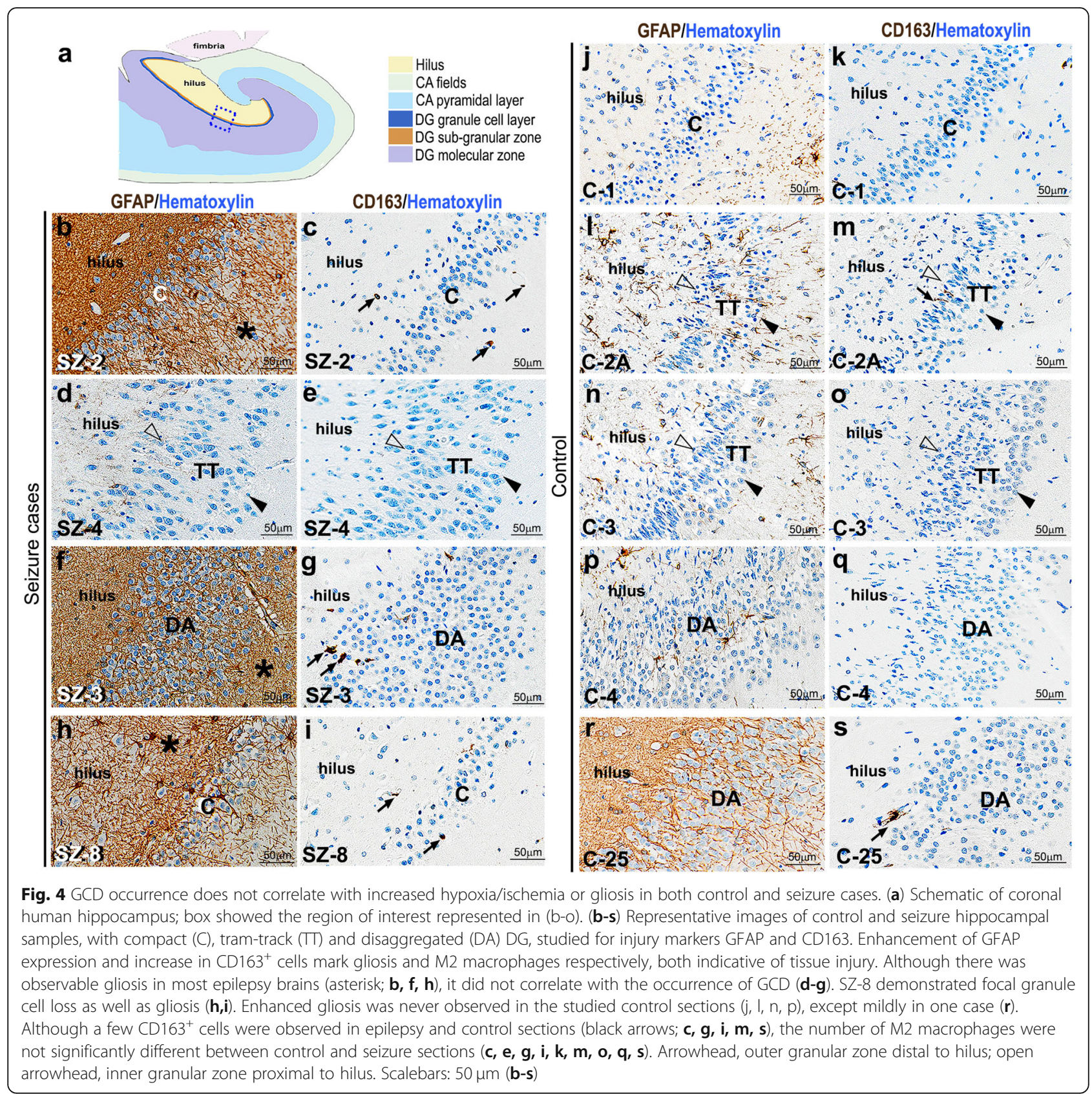

cases having or not having GCD was largely based on archived cadaveric histological slides of the hippocampus. To verify the possibility of sampling bias, we coronally sectioned one control hippocampus (C-20) at different levels across the antero-posterior axis, each section a maximum of $\sim 5 \mathrm{~mm}$ apart from the next. Contours of the hippocampus, including DG, normally change along the antero-posterior axis (Fig. 5ad). At different points along the same axis we also observed each of the morphological subtypes of the GC layer (Fig. 5e-h). The morphology of GC layer varied from overlapping compact and DA forms to prominent $\mathrm{TT}$, then a combination of $\mathrm{TT}$ and $\mathrm{DA}$ forms and back to compact forms. Although no formal morphometric analysis was performed, no correlation was suggested between anterior-posterior location or DG contour and any specific pattern of GCD. Instead, GCD appeared to be a sporadic and somewhat randomly distributed variation in DG histology.

Collectively, the data suggest that GCD is a more commonly occurring phenomenon than previously appreciated, and that over-representation of seizure patients in the hippocampal studies may relate to a more thorough 


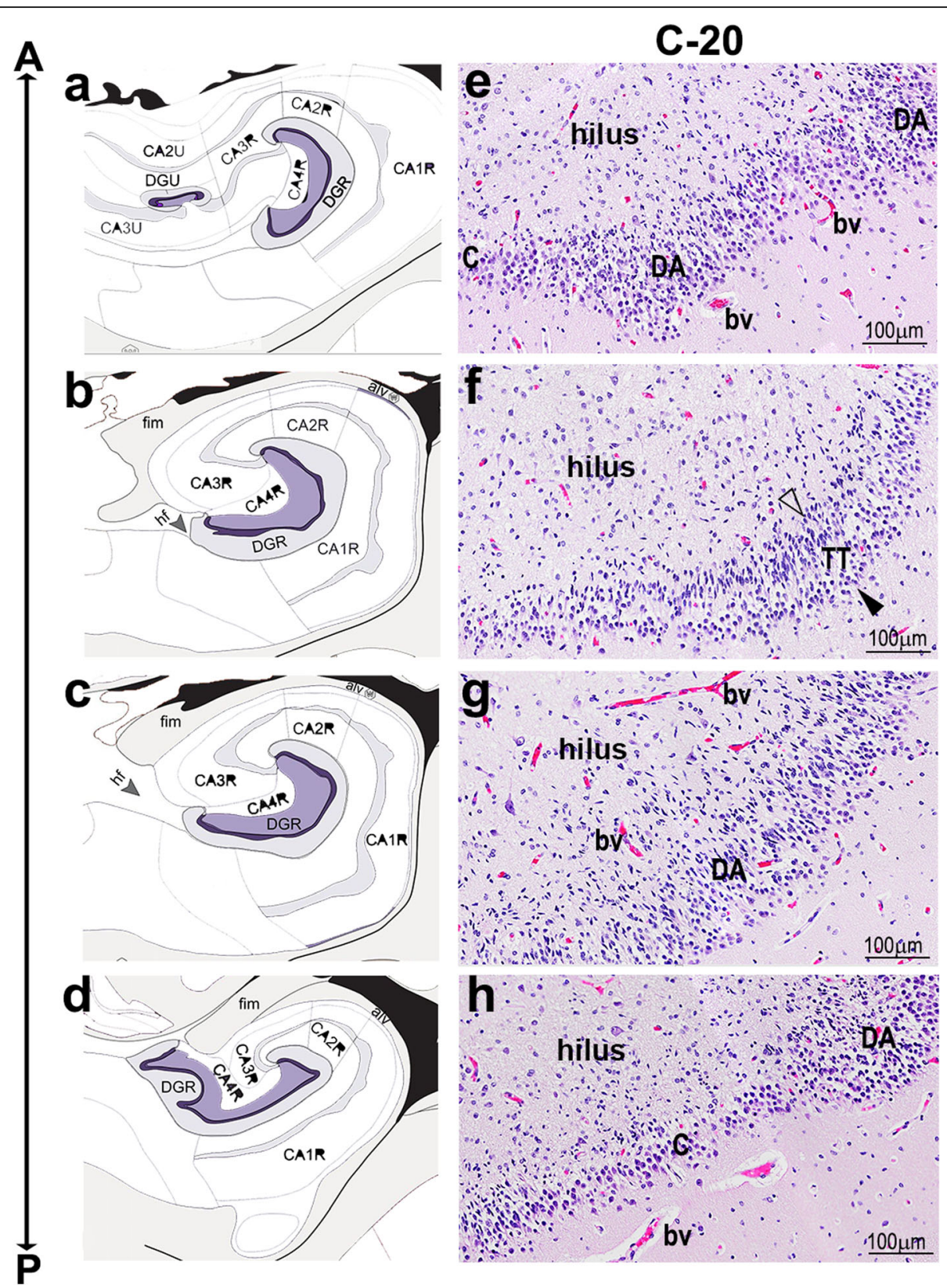

Fig. 5 GCD is not consistent across sectioning planes. (a-d) Schematics of the human hippocampus, modified from the Allen Human Brain Atlas, at different levels along the antero-posterior (A-P) axis. CA marks subdivisions of cornus ammonis (CA1-4); DG marks the dentate gyrus. The hippocampal morphology changes along the A-P axis. (e-h) Representative images of H\&E-stained coronal hippocampal sections of C-20, specifically depicting the GC layer. Sectioning plane of each section roughly corresponds to that of the adjacent schematic. The control GC layer demonstrated the entire spectrum of GCD categories: compact (C), disaggregated (DA) and tram-track (TT), often showing co-existence and repetition along the A-P axis. Arrowhead, outer granular zone distal to hilus; open arrowhead, inner granular zone proximal to hilus; bv, blood vessel. Scalebars: $100 \mu \mathrm{m}$ (e-h)

sampling in these cohorts. Most importantly, GCD is definitely not an exclusive or characteristic feature of brains affected by epilepsy.

\section{Discussion}

In this retrospective study of 147 cadaveric hippocampi from pediatric patients with seizures and controls without seizure history, we identified certain histopathological characteristics, known to be unique to epileptic brains, in both groups. We found no significant differences in neuronal morphology, molecular expression in different cell types or extent of cell loss between control and seizure-affected hippocampi. Our study challenges the existing dogma in 
neuropathology that GCD is a specific feature of chronic epilepsy, such as TLE.

\section{Defining granule cell dispersion (GCD)}

The GC layer is a prominent neuronal layer typically composed of a densely packed C-shaped band of cells in a normal hippocampus. A granule cell consists of clusters of apical dendrites, extending through the dentate molecular layer, and basal dendrites, which extend into the hilus and molecular layer in human hippocampus [4, 51]. Granule cell axons (or mossy fibers) project into the hilus to synapse with hilar interneurons, which in turn continue to synapse onto pyramidal neurons in area CA3, thus establishing part of the hippocampal circuitry. The structure and synaptic connectivity of granule cells are vital for brain functions, especially learning and memory consolidation. Ontologically, granule cells localized proximal to the outer molecular layer are born earlier than the ones found at the inner side, adjacent to the hilus [7, $45,50]$. Pathologically, this cell layer is found to be susceptible to hippocampal insults, injury, hypoxia, brain malformations and epilepsy [15, 23, 29]. These conditions often lead to hippocampal sclerosis characterized by neuronal cell loss and reactive gliosis, microglial infiltration and, according to prior studies, GCD $[10,54]$.

GCD is a histological phenotype reported in the affected dentate gyri of patients suffering from chronic epilepsy $[3,5,10,15,30,55,56]$. GCD has been described in $\sim 40 \%$ of the hippocampi of TLE patients [9, 56, 59]. Similar to the human epilepsy-affected brains, animal models of epilepsy demonstrated different kinds of dispersed GC layer, thought to be replicative of human patient pathophysiology [40, 43]. Variable overlapping patterns of GCD have been described. These include focal dispersed clusters of granule cells ectopically present in either hilus or molecular DG layer, segmental broadening and duplication or "tram-tracking" of the GC layer [8, 21, 30]. Some reports categorized bilamination separately from GCD [56]. In our study, both focal broadening and bilamination of GC layer were considered as subtypes of GCD.

Different approaches also exist with regard to the assessment of what should be considered as "GCD". In our study, we have adapted and modified the GCD classification as proposed by Blumcke et al. [8], where the presence of different grades of GCD was subjectively assessed, primarily based on differential GC density in the DG (focal broadening), blurring of the boundary or ectopic presence of clusters or bilayer of granule cells. Definition of GCD in this manner can be easily translated to routine surgical pathology practice, as it requires no special stains, image analysis or laborious cell counts.
Not surprisingly, some variation of the Blumcke et al. (2009) definition has been used in many published studies of GCD and its putative specific association with epilepsy; however, most of these either lacked or included only a few non-seizure controls for comparison (Supplementary Table 2, Additional File 1). Further, we noted that alternative methods of GCD assessment exist. Since dispersed GC layer is generally thicker than "normal", some studies assessed GCD based on a morphometric method of averaging measurements of GC layer thickness across "straight" parts of DG and then re-averaging values obtained from several sections per hippocampus $[24,37,52]$. In these studies, GCD was defined as an average DG thickness whose value measured greater than a range of average thicknesses obtained from nonseizure controls. As illustrated in Supplementary Fig. 6 (in Additional File 1), this approach, though quantitative, is likely to exclude focal dispersion, which is specifically associated with epilepsy in many publications. Although the morphometric approach may seem less subjective than our study design, determination of what constitutes a "straight" segment of the DG is also highly subjective and may bias against inclusion of focal GCD in these analyses.

\section{GCD is not exclusively present in seizure-affected brains}

Although many reports have accepted the correlative dogma of presence of GCD with seizure history, contradictions exist in the field. One study suggests that GCD is more correlated with learning and memory changes than to seizures, hippocampal sclerosis or neuronal loss [8]. GCD has also been observed in many children, with no history of seizures, who died suddenly and unexpectedly [26, 31, 32]. Although the hippocampal findings in the latter research were interpreted as potentially significant malformations, the possibility of normal variation was not excluded. Another retrospective study of 68 SUDEP (sudden unexpected death in epilepsy) cases showed no evidence of significant differences in hippocampal position or shape or GC abnormalities compared to 53 age-matched non-epilepsy controls, although neither DA nor TT were specifically evaluated [52]. Similarly, a recent study concerning sudden unexplained death in childhood (SUDC) cases also could not clearly relate hippocampal abnormalities to either cause or effect of seizures [39]. We are aware of only one report mentioning the existence of GCD in human patients with no history of epilepsy or seizures [25]. This study reported bilateral GCD in 3 post-mortem pediatric cases, of which only 1 had epilepsy. GCD in the non-seizure brain samples included both DA and TT subtypes, as defined in our study. We also observed GCD in the published images of developing normal human hippocampus, at GW20 and GW 23-25 [12]. However, 
most human studies, using either surgical resection samples or autopsied hippocampi, lacked substantial number or age-matched controls for a statistically robust comparison.

To develop a comprehensive, comparative study of human hippocampal histopathology, with or without the clinical history of seizures, we performed a retrospective survey of 147 cadaveric cases, with 126 controls (no history of seizure) and 21 cases with clinical history of epilepsy. Our studied cohort is limited to a hospital-based autopsy series of largely pediatric patients, each of whom was diagnosed with one or more comorbidities. We did not have access to "true normal controls", such as deaths due to sudden trauma or non-hospital based forensic cases. Moreover, being a retrospective study, we did not have control over the postmortem interval to autopsy or brain fixation period, variables that might affect certain molecular analyses. We were also limited to clinical information available in the patients' medical records. It was impossible to exclude the possibility that some of the controls had undocumented non-convulsive or convulsive seizures or were destined to develop clinical epilepsy had they survived.

In our study, we identified the entire spectrum of GCD in the control dentate gyri, similar in frequency and morphologically indistinct from those observed in the seizure population. This study is the first of its kind to identify GCD in such a large set of human brain samples with no history of seizures. The high prevalence of GCD among the controls in this study and absence of correlation with age, gender, PMI or clinicalpathological diagnoses collectively suggest that GCD, as presently defined, is a variation in normal hippocampal microanatomy and should not be considered as a specific or selective feature of any form of chronic epilepsy, including TLE. Moreover, cell loss was not overtly observed in most of our hippocampal samples, irrespective of GCD, similar to previous report [25]. Thus, our study re-emphasizes the fact that GCD is not a mere consequence of loss of intervening neurons.

\section{Similar molecular expression patterns in dispersed GC layers of both control and seizure-affected brains raise doubts about putative pathogenesis of GCD}

To understand the basis of GCD, a few mechanisms have been proposed using both mouse models and human brain samples affected by epilepsy. In mouse models of febrile and chemical-induced seizures, aberrant migration of either new immature neurons or differentiated mature granule cells were correlated with the occurrence of GCD under the influence of seizures [11, 33, 40]. Defects in GC migration have also been suggested as the basis for GCD in human hippocampi influenced by epileptogenesis [30, 54]. Alternate putative mechanisms include severe cell loss, gliosis/gliogenesis, ectopic sprouting of mossy fibers and abnormal expression of growth factors [15, 35, 37]. Reelin is an important signaling molecule, known to control GC migration by maintaining the radial glial scaffold [22]. Biochemical and functional studies in model systems confirm that formation of a densely packed GC layer is dependent on proper Reelin signaling [58, 62]. Further, human studies as well as genetic and chemical-induced mouse models of epilepsy showed association between reduced Reelin mRNA expression and GCD [13, 16, 22, 24, 27, 42]; although there is discrepancy in the type of Reelin ${ }^{+}$cells (early-born Cajal-Retzius cells or late-born hilar interneurons) considered to be associated with GCD. Introduction of exogenous Reelin into kainate-injected mouse hippocampus was also reported to prevent GCD in mice [41]. However, regardless of whether seizure-related disruption of Reelin signaling, or other factors, influence DG compaction in humans, the morphological consequences of such perturbation with regard to GCD did not appear to be distinguishable from many control hippocampi.

In our retrospective study, we did not observe an overt increase in cell loss or in the number of activated macrophage and microglia $\left(\mathrm{CD}_{163}{ }^{+}, \mathrm{CD} 8^{+}\right.$, $\mathrm{IBA}^{+}$cells), in either control or seizure-affected hippocampi. Although hippocampi of many epilepsy patients demonstrate DG and/or hilus infiltration by $\mathrm{GFAP}^{+}$astrocytes indicative of injury-driven gliosis, this alteration was absent in 1 of the 4 seizureaffected hippocampi and in all 4 control brain samples with GCD that we tested. Contradictory reports also debate the association of GCD with seizureinduced cell proliferation in the human hippocampus $[20,55]$. In our study, no enhancement of neural progenitor markers (SOX2, PAX6, TBR2) was observed in either control or epileptic brains, with or without GCD. Furthermore, we noted prominent expression of Calbindin and CTIP2 in the outer GC layer of both control and seizure cases with "tram-track" GCD. This expression pattern is similar to that seen in normal dentate gyri $[1,12]$, indicating concentrated presence of mature neurons in the outer GC layer. Such differential expression was however not observed in the "disaggregated" subtype of GCD in control and seizure brains. This suggests that unlike the "tramtrack" GCD, the molecular or anatomical distinction between inner and outer GC layers may largely be blurred in the controls and epilepsy brains with "disaggregated" GCD. In summary, these data largely suggest that parameters like cell loss, enhanced proliferation, abnormal neuronal layering, gliosis or microglia/macrophage invasion cannot distinguish GCD between controls and seizure-affected brains, 
and hence cannot be considered commonly responsible for GCD. Rather, we suspect that GCD represents normal variation in the microanatomic DG structure, which may well be present from or before birth, and have no clinical significance.

\section{Potential influence of sampling bias on GCD incidence in epilepsy patients}

While analyzing multiple sections from the same hippocampal block of control and epilepsy patients for histochemical studies, we encountered intra-hippocampal differences in the extent and severity of the GCD phenotypes. To understand this issue more systematically, we made paraffin blocks of a control cadaveric human hippocampus along the entire antero-posterior length and analyzed multiple representative sections from each block. Intriguingly, we found that the morphology of the hippocampal GC layer varied frequently (within 2-3 mm) and extensively, ranging from compact to disaggregated to bilaminar forms, and also demonstrated combinations of GCD subtypes. The patchy nature of GCD within hippocampi may have contributed to the misconception that GCD is more common in epileptics, particularly those who underwent surgical resection of their hippocampi for TLE. It is very likely that pathologists, and especially researchers, have examined multiple histological sections from the hippocampi of these patients, as opposed to the single hippocampal section evaluated as part of routine autopsy. For example, such variation in the severity of GCD across the antero-posterior axis within a specimen was marked in $23 \%$ of the epileptic surgical resection cases studied in [56]. It may be primarily because of the large number of control hippocampi examined in this study with specific attention to DG histology that the fallacy of GCD as a seizurespecific pathological phenotype became evident. We also predict that the percentage of GCD in controls, obtained predominantly by analyzing one autopsy section, may be underrepresented.

\section{Conclusions}

This retrospective human hippocampal survey is the first study to report GCD in a large number of patients without the clinical history of epilepsy. We found no direct association of GCD with seizure or other clinical history, age of death, post-mortem interval or gender. Morphological, histochemical and statistical tests confirmed no overt differences in GCD between control and seizureaffected brains. Most significantly, we determined that the entire morphological spectrum of GC layer exists across the varying hippocampal planes, both in control and epileptic brains. This suggests that the phenomenon of granule cell dispersion is likely a variation of normal hippocampal anatomy that should not be considered a cause or consequence of epilepsy.

\section{Supplementary information}

Supplementary information accompanies this paper at https://doi.org/10. 1186/s40478-020-00928-3.

\section{Additional file 1. Supplementary Tables and Figures. \\ Supplementary Table 1: List of patients evaluated with}

immunohistochemistry/immunofluorescence; Supplementary Table 2:

List of seizure patients and controls in published reports of GCD;

Supplementary Fig. 1: Control hippocampi demonstrate variable

severities of GCD; Supplementary Fig. 2: GCD occurs independent of

different clinical parameters in patients with or without epileptic history;

Supplementary Fig. 3: Distribution of neural progenitors is similar in

cadaveric controls and seizure cases with GCD; Supplementary Fig. 4:

Cadaveric control and seizure brains do not show differential activation

of macrophages and microglia; Supplementary Fig. 5: GCD severity

varies across sectioning planes in both control and seizure brains;

Supplementary Fig. 6: Alternative assessment method for GCD -

averaging across measurements fails to resolve focal forms of GCD.

Additional file 2. Full list of cases with clinical history. List of total

147 autopsy cases studied, along with a table of their clinical history obtained from the patient records and histology archives of Seattle Children's Hospital Department of Pathology (2014-2019). Variables analyzed included age, gender, post-mortem interval, seizure history and interval (onset till death) and major systemic clinical diagnoses including malformations or other problems in central nervous system (CNS), which denotes many acquired forms of CNS injury such as hypoxic-ischemic encephalopathy (HIE), cerebral edema. The blue highlights demarcate the seizure/epilepsy cases tabulated in Table 1. The pink highlights

demarcate the controls demonstrating GCD, as tabulated inhippocampal section. GW, gestational weeks; TT, tram-track; DA, disaggregated; PMI, post-mortem interval; hr, hour(s); wk, week(s); mo, month(s); yr, year(s). Table 2. The non-highlighted rows demarcate cadaveric control reports with no clinical history of seizures and no evidence of GCD in any available hippocampal section. GW, gestational weeks; $\Pi$, tram-track; DA, disaggregated; PMI, post-mortem interval; hr, hour(s); wk, week(s); mo, month(s); yr, year(s).

\section{Abbreviations}

C: Compact; C-\#: "Control" patient sample; DA: Disaggregated; DG: Dentate gyrus; GC: Granule cell; GCD: Granule cell dispersion; GW: Gestation weeks; h: Hour(s); PMI: Post-mortem interval; SZ-\#: "Seizure" patient sample; TLE: Temporal lobe epilepsy; TT: Tram-track

\section{Acknowledgements}

Schematics of human hippocampi from the Allen Human Brain Atlas were obtained and modified as per the requirement of this paper (Fig. 5). We thank the Seattle Children's Hospital archives for the specimens and clinical records.

\section{Authors' contributions}

AR, RPK contributed to the study conception and design. AR contributed to the data collection and statistical analyses. AR, KJM, RPK contributed to the interpretation of the data. KJM provided the funding resources. AR wrote the first draft of the manuscript and all authors commented on previous versions of the manuscript. All authors read and approved the final manuscript.

\section{Funding}

This work is funded by the NIH grant 1R01NS099027 (KJM).

Availability of data and materials

All data generated or analyzed during this study are included in this article and its supplementary information files.

Ethics approval and consent to participate

This study only used hippocampal sections and paraffin blocks of human cadavers, obtained from the archives of Seattle Children's Hospital, 
Department of Pathology. Since this study was completely based on cadaveric brain samples and relevant clinical history, the Institutional Review Board (IRB) of Seattle Children's Hospital determined that this was not research involving human subjects, and hence was exempted from full IRB review and approval.

\section{Consent for publication}

Not applicable.

\section{Competing interests}

The authors declare that they have no competing interests.

\section{Author details}

${ }^{1}$ Center for Integrative Brain Research, Seattle Children's Research Institute, Seattle, Washington, USA. ²Division of Genetic Medicine, Department of Pediatrics, University of Washington, Seattle, Washington, USA. ${ }^{3}$ Department of Pathology, Seattle Children's Hospital, Seattle, Washington, USA. ${ }^{4}$ Division of Pathology, University of Washington, Seattle, Washington, USA.

\section{Received: 10 February 2020 Accepted: 3 April 2020}

Published online: 21 April 2020

\section{References}

1. Abraham H, Richter Z, Gyimesi C, Horvath Z, Janszky J, Doczi T, Seress L (2011) Degree and pattern of calbindin immunoreactivity in granule cells of the dentate gyrus differ in mesial temporal sclerosis, cortical malformationand tumor-related epilepsies. Brain Res 1399:66-78. https://doi.org/10.1016/j. brainres.2011.05.010

2. Abraham H, Veszpremi B, Kravjak A, Kovacs K, Gomori E, Seress L (2009) Ontogeny of calbindin immunoreactivity in the human hippocampal formation with a special emphasis on granule cells of the dentate gyrus. Int J Dev Neurosci 27:115-127. https://doi.org/10.1016/j.ijdevneu.2008.12.004

3. Alcantara D, Timms AE, Gripp K, Baker L, Park K, Collins S, Cheng C, Stewart F, Mehta SG, Saggar A, Sztriha L, Zombor M, Caluseriu O, Mesterman R, Van Allen MI, Jacquinet A, Ygberg S, Bernstein JA, Wenger AM, Guturu H, Bejerano G, Gomez-Ospina N, Lehman A, Alfei E, Pantaleoni C, Conti V, Guerrini R, Moog U, Graham JM Jr, Hevner R, Dobyns WB, O'Driscoll M, Mirzaa GM (2017) Mutations of AKT3 are associated with a wide spectrum of developmental disorders including extreme megalencephaly. Brain 140: 2610-2622. https://doi.org/10.1093/brain/awx203

4. Amaral DG, Scharfman HE, Lavenex P (2007) The dentate gyrus: fundamental neuroanatomical organization (dentate gyrus for dummies). Prog Brain Res 163:3-22. https://doi.org/10.1016/S0079-6123(07)63001-5

5. Armstrong DD (1993) The neuropathology of temporal lobe epilepsy. J Neuropathol Exp Neurol 52:433-443

6. Bae EK, Jung KH, Chu K, Lee ST, Kim JH, Park KI, Kim M, Chung CK, Lee SK Roh JK (2010) Neuropathologic and clinical features of human medial temporal lobe epilepsy. J Clin Neurol 6:73-80. https://doi.org/10.3988/jcn. 2010.6.2.73

7. Bayer SA, Altman J (1974) Hippocampal development in the rat: cytogenesis and morphogenesis examined with autoradiography and low-level Xirradiation. J Comp Neurol 158:55-79. https://doi.org/10.1002/cne.901580105

8. Blumcke I, Kistner I, Clusmann H, Schramm J, Becker AJ, Elger CE, Bien CG, Merschhemke M, Meencke HJ, Lehmann T, Buchfelder M, Weigel D, Buslei R, Stefan H, Pauli E, Hildebrandt M (2009) Towards a clinico-pathological classification of granule cell dispersion in human mesial temporal lobe epilepsies. Acta Neuropathol 117:535-544. https://doi.org/10.1007/s00401009-0512-5

9. Blumcke I, Pauli E, Clusmann H, Schramm J, Becker A, Elger C, Merschhemke M, Meencke HJ, Lehmann T, von Deimling A, Scheiwe C, Zentner J, Volk B, Romstock J, Stefan H, Hildebrandt M (2007) A new clinico-pathological classification system for mesial temporal sclerosis. Acta Neuropathol 113: 235-244. https://doi.org/10.1007/s00401-006-0187-0

10. Caboclo LO, Neves RS, Jardim AP, Hamad AP, Centeno RS, Lancellotti CL, Scorza CA, Cavalheiro EA, Yacubian EM, Sakamoto AC (2012) Surgical and postmortem pathology studies: contribution for the investigation of temporal lobe epilepsy. Arq Neuropsiquiatr 70:945-952. https://doi.org/10. 1590/s0004-282x2012001200009

11. Chai X, Munzner G, Zhao S, Tinnes S, Kowalski J, Haussler U, Young C, Haas CA, Frotscher M (2014) Epilepsy-induced motility of differentiated neurons. Cereb Cortex 24:2130-2140. https://doi.org/10.1093/cercor/bht067
12. Cipriani S, Journiac N, Nardelli J, Verney C, Delezoide AL, Guimiot F, Gressens P, Adle-Biassette H (2017) Dynamic expression patterns of progenitor and neuron layer markers in the developing human dentate Gyrus and fimbria. Cereb Cortex 27:358-372. https://doi.org/10.1093/cercor/ bhv223

13. D'Arcangelo G, Miao GG, Chen SC, Soares HD, Morgan Jl, Curran T (1995) A protein related to extracellular matrix proteins deleted in the mouse mutant reeler. Nature 374:719-723. https://doi.org/10.1038/374719a0

14. Davies KG, Hermann BP, Dohan FC Jr, Foley KT, Bush AJ, Wyler AR (1996) Relationship of hippocampal sclerosis to duration and age of onset of epilepsy, and childhood febrile seizures in temporal lobectomy patients. Epilepsy Res 24:119-126. https://doi.org/10.1016/0920-1211(96)00008-3

15. de Lanerolle NC, Lee TS, Spencer DD. Histopathology of Human Epilepsy. In: Noebels JL, Avoli M, Rogawski MA, et al., editors. Jasper's Basic Mechanisms of the Epilepsies [Internet]. 4th edition. Bethesda (MD): National Center for Biotechnology Information (US); 2012. Available from: https://www.ncbi.nIm. nih.gov/books/NBK98141/

16. Duveau V, Madhusudan A, Caleo M, Knuesel I, Fritschy JM (2011) Impaired reelin processing and secretion by Cajal-Retzius cells contributes to granule cell dispersion in a mouse model of temporal lobe epilepsy. Hippocampus 21:935-944. https://doi.org/10.1002/hipo.20793

17. El Bahh B, Lespinet V, Lurton D, Coussemacq M, Le Gal La Salle G, Rougier A (1999) Correlations between granule cell dispersion, mossy fiber sprouting, and hippocampal cell loss in temporal lobe epilepsy. Epilepsia 40:13931401. https://doi.org/10.1111/j.1528-1157.1999.tb02011.x

18. Eng LF, Ghirnikar RS (1994) GFAP and astrogliosis. Brain Pathol 4:229-237. https://doi.org/10.1111/j.1750-3639.1994.tb00838.x

19. Etzerodt A, Moestrup SK (2013) CD163 and inflammation: biological, diagnostic, and therapeutic aspects. Antioxid Redox Signal 18:2352-2363. https://doi.org/10.1089/ars.2012.4834

20. Fahrner A, Kann G, Flubacher A, Heinrich C, Freiman TM, Zentner J, Frotscher M, Haas CA (2007) Granule cell dispersion is not accompanied by enhanced neurogenesis in temporal lobe epilepsy patients. Exp Neurol 203: 320-332. https://doi.org/10.1016/j.expneurol.2006.08.023

21. Freiman TM, Eismann-Schweimler J, Frotscher M (2011) Granule cell dispersion in temporal lobe epilepsy is associated with changes in dendritic orientation and spine distribution. Exp Neurol 229:332-338. https://doi.org/ 10.1016/j.expneurol.2011.02.017

22. Frotscher M, Haas CA, Forster E (2003) Reelin controls granule cell migration in the dentate gyrus by acting on the radial glial scaffold. Cereb Cortex 13: 634-640. https://doi.org/10.1093/cercor/13.6.634

23. Girgis F, Pace J, Sweet J, Miller JP (2016) Hippocampal neurophysiologic changes after mild traumatic brain injury and potential Neuromodulation treatment approaches. Front Syst Neurosci 10:8. https://doi.org/10.3389/ fnsys.2016.00008

24. Haas CA, Dudeck O, Kirsch M, Huszka C, Kann G, Pollak S, Zentner J, Frotscher M (2002) Role for reelin in the development of granule cell dispersion in temporal lobe epilepsy. J Neurosci 22:5797-5802 Doi:20026621

25. Harding B, Thom M (2001) Bilateral hippocampal granule cell dispersion: autopsy study of 3 infants. Neuropathol Appl Neurobiol 27:245-251

26. Hefti MM, Cryan JB, Haas EA, Chadwick AE, Crandall LA, Trachtenberg FL, Armstrong DD, Grafe M, Krous HF, Kinney HC (2016) Hippocampal malformation associated with sudden death in early childhood: a neuropathologic study: part 2 of the investigations of the San Diego SUDC research project. Forensic Sci Med Pathol 12:14-25. https://doi.org/10.1007/ s12024-015-9731-3

27. Heinrich C, Nitta N, Flubacher A, Muller M, Fahrner A, Kirsch M, Freiman T, Suzuki F, Depaulis A, Frotscher M, Haas CA (2006) Reelin deficiency and displacement of mature neurons, but not neurogenesis, underlie the formation of granule cell dispersion in the epileptic hippocampus. J Neurosci 26:4701-4713. https://doi.org/10.1523/JNEUROSC1.5516-05.2006

28. Hendrickx DAE, van Eden CG, Schuurman KG, Hamann J, Huitinga I (2017) Staining of HLA-DR, Iba1 and CD68 in human microglia reveals partially overlapping expression depending on cellular morphology and pathology. J Neuroimmunol 309:12-22. https://doi.org/10.1016/j.jneuroim.2017.04.007

29. Hossmann KA (1999) The hypoxic brain. Insights from ischemia research. Adv Exp Med Biol 474:155-169

30. Houser CR (1990) Granule cell dispersion in the dentate gyrus of humans with temporal lobe epilepsy. Brain Res 535:195-204

31. Kinney HC, Cryan JB, Haynes RL, Paterson DS, Haas EA, Mena OJ, Minter M, Journey KW, Trachtenberg FL, Goldstein RD, Armstrong DD (2015) Dentate 
gyrus abnormalities in sudden unexplained death in infants: morphological marker of underlying brain vulnerability. Acta Neuropathol 129:65-80. https://doi.org/10.1007/s00401-014-1357-0

32. Kinney HC, Hefti MM, Goldstein RD, Haynes RL (2018) Sudden infant death syndrome. In developmental neuropathology. In: Adle-Biassette H, Harding BN, Golden J (eds) Developmental neuropathology. https://doi.org/10.1002/ 9781119013112.ch25

33. Koyama R, Tao K, Sasaki T, Ichikawa J, Miyamoto D, Muramatsu R, Matsuki N, Ikegaya $Y$ (2012) GABAergic excitation after febrile seizures induces ectopic granule cells and adult epilepsy. Nat Med 18:1271-1278. https://doi.org/10. $1038 / \mathrm{nm} .2850$

34. Lavado A, Oliver G (2007) Prox1 expression patterns in the developing and adult murine brain. Dev Dynamics 236:518-524. https://doi.org/10.1002/ dvdy.21024

35. Lurton D, Sundstrom L, Brana C, Bloch B, Rougier A (1997) Possible mechanisms inducing granule cell dispersion in humans with temporal lobe epilepsy. Epilepsy Res 26:351-361. https://doi.org/10.1016/s09201211(96)01002-9

36. Marucci G, Rubboli G, Giulioni M (2010) Role of dentate gyrus alterations in mesial temporal sclerosis. Clin Neuropathol 29:32-35. https://doi.org/10. 5414/npp29032

37. Mathern GW, Kuhlman PA, Mendoza D, Pretorius JK (1997) Human fascia dentata anatomy and hippocampal neuron densities differ depending on the epileptic syndrome and age at first seizure. J Neuropathol Exp Neurol 56:199-212. https://doi.org/10.1097/00005072-199702000-00011

38. Matsue K, Minakawa S, Kashiwagi T, Toda K, Sato T, Shioda S, Seki T (2018) Dentate granule progenitor cell properties are rapidly altered soon after birth. Brain Struct Funct 223:357-369. https://doi.org/10.1007/s00429-017-1499-7

39. McGuone D, Leitner D, William C, Faustin A, Leelatian N, Reichard R, Shepherd TM, Snuderl M, Crandall L, Wisniewski T, Devinsky O (2020) Neuropathologic changes in sudden unexplained death in childhood. J Neuropathol Exp Neurol. https://doi.org/10.1093/jnen/nlz136

40. Mello LE, Cavalheiro EA, Tan AM, Pretorius JK, Babb TL, Finch DM (1992) Granule cell dispersion in relation to mossy fiber sprouting, hippocampal cell loss, silent period and seizure frequency in the pilocarpine model of epilepsy. Epilepsy Res Suppl 9:51-59 discussion 59-60

41. Muller MC, Osswald M, Tinnes S, Haussler U, Jacobi A, Forster E, Frotscher M, Haas CA (2009) Exogenous reelin prevents granule cell dispersion in experimental epilepsy. Exp Neurol 216:390-397. https://doi.org/10.1016/j. expneurol.2008.12.029

42. Orcinha C, Munzner G, Gerlach J, Kilias A, Follo M, Egert U, Haas CA (2016) Seizure-induced motility of differentiated dentate granule cells is prevented by the central Reelin fragment. Front Cell Neurosci 10:183. https://doi.org/ 10.3389/fncel.2016.00183

43. Parent JM, Yu TW, Leibowitz RT, Geschwind DH, Sloviter RS, Lowenstein DH (1997) Dentate granule cell neurogenesis is increased by seizures and contributes to aberrant network reorganization in the adult rat hippocampus. J Neurosci 17:3727-3738

44. Prayson RA, Cohen ML (2000) Practical differential diagnosis in surgical neuropathology, 1st edn. Humana Press. https://doi.org/10.1007/978-159259-037-7

45. Rakic P, Nowakowski RS (1981) The time of origin of neurons in the hippocampal region of the rhesus monkey. J Comp Neurol 196:99-128. https://doi.org/10.1002/cne.901960109

46. Riviere JB, Mirzaa GM, O'Roak BJ, Beddaoui M, Alcantara D, Conway RL, StOnge J, Schwartzentruber JA, Gripp KW, Nikkel SM, Worthylake T, Sullivan CT, Ward TR, Butler HE, Kramer NA, Albrecht B, Armour CM, Armstrong L, Caluseriu O, Cytrynbaum C, Drolet BA, Innes AM, Lauzon JL, Lin AE, Mancini GM, Meschino WS, Reggin JD, Saggar AK, Lerman-Sagie T, Uyanik G, Weksberg R, Zirn B, Beaulieu CL, Majewski J, Bulman DE, O'Driscoll M, Shendure J, Graham JM Jr, Boycott KM, Dobyns WB (2012) De novo germline and postzygotic mutations in AKT3, PIK3R2 and PIK3CA cause a spectrum of related megalencephaly syndromes. Nat Genet 44:934-940. https://doi.org/10.1038/ng.2331

47. Roy A, Murphy RM, Deng M, MacDonald JW, Bammler TK, Aldinger KA, Glass IA, Millen KJ (2019) PI3K-yap activity drives cortical gyrification and hydrocephalus in mice. eLife 8:e45961. https://doi.org/10.7554/eLife.45961

48. Roy A, Skibo J, Kalume F, Ni J, Rankin S, Lu Y, Dobyns WB, Mills GB, Zhao JJ, Baker SJ, Millen KJ (2015) Mouse models of human PIK3CA-related brain overgrowth have acutely treatable epilepsy. eLife 4:e12703. https://doi.org/10.7554/eLife.12703
49. Seress $L$ (1992) Morphological variability and developmental aspects of monkey and human granule cells: differences between the rodent and primate dentate gyrus. Epilepsy Res Suppl 7:3-28

50. Seress L, Abraham H, Tornoczky T, Kosztolanyi G (2001) Cell formation in the human hippocampal formation from mid-gestation to the late postnatal period. Neuroscience 105:831-843. https://doi.org/10.1016/s03064522(01)00156-7

51. Seress L, Mrzljak L (1987) Basal dendrites of granule cells are normal features of the fetal and adult dentate gyrus of both monkey and human hippocampal formations. Brain Res 405:169-174. https://doi.org/10.1016/ 0006-8993(87)91003-1

52. Somani A, Zborovschi AB, Liu Y, Patodia S, Michalak Z, Sisodiya SM, Thom M (2019) Hippocampal morphometry in sudden and unexpected death in epilepsy. Neurology 93:e804-e814. https://doi.org/10.1212/WNL. 0000000000007969

53. Sorrells SF, Paredes MF, Cebrian-Silla A, Sandoval K, Qi D, Kelley KW, James D, Mayer S, Chang J, Auguste Kl, Chang EF, Gutierrez AJ, Kriegstein AR, Mathern GW, Oldham MC, Huang EJ, Garcia-Verdugo JM, Yang Z, AlvarezBuylla A (2018) Human hippocampal neurogenesis drops sharply in children to undetectable levels in adults. Nature 555:377-381. https://doi.org/10. 1038/nature25975

54. Thom M (2014) Review: hippocampal sclerosis in epilepsy: a neuropathology review. Neuropathol Appl Neurobiol 40:520-543. https:// doi.org/10.1111/nan.12150

55. Thom M, Martinian L, Williams G, Stoeber K, Sisodiya SM (2005) Cell proliferation and granule cell dispersion in human hippocampal sclerosis. J Neuropathol Exp Neurol 64:194-201. https://doi.org/10.1093/jnen/64.3.194

56. Thom M, Sisodiya SM, Beckett A, Martinian L, Lin WR, Harkness W, Mitchell TN, Craig J, Duncan J, Scaravilli F (2002) Cytoarchitectural abnormalities in hippocampal sclerosis. J Neuropathol Exp Neurol 61:510-519. https://doi. org/10.1093/jnen/61.6.510

57. Turtzo LC, Lescher J, Janes L, Dean DD, Budde MD, Frank JA (2014) Macrophagic and microglial responses after focal traumatic brain injury in the female rat. J Neuroinflammation 11:82. https://doi.org/10.1186/1742-2094-11-82

58. Weiss KH, Johanssen C, Tielsch A, Herz J, Deller T, Frotscher M, Forster E (2003) Malformation of the radial glial scaffold in the dentate gyrus of reeler mice, scrambler mice, and ApoER2/NLDLR-deficient mice. J Comp Neurol 460:56-65. https://doi.org/10.1002/cne.10644

59. Wieser HG, Epilepsy ICoNo (2004) ILAE commission report. Mesial temporal lobe epilepsy with hippocampal sclerosis. Epilepsia 45:695-714. https://doi. org/10.1111/j.0013-9580.2004.09004.x

60. Yamawaki R, Thind K, Buckmaster PS (2015) Blockade of excitatory synaptogenesis with proximal dendrites of dentate granule cells following rapamycin treatment in a mouse model of temporal lobe epilepsy. J Comp Neurol 523:281-297. https://doi.org/10.1002/cne.23681

61. Yu DX, Marchetto MC, Gage FH (2014) How to make a hippocampal dentate gyrus granule neuron. Development 141:2366-2375. https://doi.org/ 10.1242/dev.096776

62. Zhao S, Chai X, Forster E, Frotscher M (2004) Reelin is a positional signal for the lamination of dentate granule cells. Development 131:5117-5125. https://doi.org/10.1242/dev.01387

\section{Publisher's Note}

Springer Nature remains neutral with regard to jurisdictional claims in published maps and institutional affiliations.

Ready to submit your research? Choose BMC and benefit from:

- fast, convenient online submission

- thorough peer review by experienced researchers in your field

- rapid publication on acceptance

- support for research data, including large and complex data types

- gold Open Access which fosters wider collaboration and increased citations

- maximum visibility for your research: over $100 \mathrm{M}$ website views per year

At $\mathrm{BMC}$, research is always in progress.

Learn more biomedcentral.com/submissions 